\title{
Fusion categories in terms of graphs and relations
}

\author{
Hendryk Pfeiffer
}

\begin{abstract}
Every fusion category $C$ that is $k$-linear over a suitable field $k$, is the category of finite-dimensional comodules of a weak Hopf algebra $H$. This weak Hopf algebra is finite-dimensional, cosemisimple and has commutative bases. It arises as the universal coend with respect to the long canonical functor $\omega: \mathcal{C} \rightarrow$ Vect $_{k}$. We show that $H$ is a quotient $H=H[\mathscr{E}] / I$ of a weak bialgebra $H[\mathscr{E}]$ which has a combinatorial description in terms of a finite directed graph $\mathcal{E}$ that depends on the choice of a generator $M$ of $\mathcal{C}$ and on the fusion coefficients of $\mathscr{C}$. The algebra underlying $H[\mathscr{E}]$ is the path algebra of the quiver $\mathcal{E} \times \mathcal{E}$, and so the composability of paths in $\mathcal{E}$ parameterizes the truncation of the tensor product of $\mathcal{C}$. The ideal $I$ is generated by two types of relations. The first type enforces that the tensor powers of the generator $M$ have the appropriate endomorphism algebras, thus providing a Schur-Weyl dual description of $\mathcal{C}$. If $\mathcal{C}$ is braided, this includes relations of the form ' $R T T=T T R$ ' where $R$ contains the coefficients of the braiding on $\omega M \otimes \omega M$, a generalization of the construction of Faddeev-Reshetikhin-Takhtajan to weak bialgebras. The second type of relations removes a suitable set of group-like elements in order to make the category of finite-dimensional comodules equivalent to $\mathcal{C}$ over all tensor powers of the generator $M$. As examples, we treat the modular categories associated with $U_{q}\left(\mathfrak{s l}_{2}\right)$.
\end{abstract}

Mathematics Subject Classification (2010). 16W30, 18D10.

Keywords. Fusion category, braided monoidal category, weak Hopf algebra, Tannaka-Kreĭn reconstruction, quiver.

\section{Contents}

1 Introduction . . . . . . . . . . . . . . . . . . . . . . . 340

2 Preliminaries. . . . . . . . . . . . . . . . . 342

3 A combinatorial cover of the universal coend . . . . . . . . . . 350

4 Schur-Weyl dual description at fixed powers of $M \ldots \ldots$. . . . . . 354

5 Comparing different powers of $M \ldots \ldots \ldots \ldots$

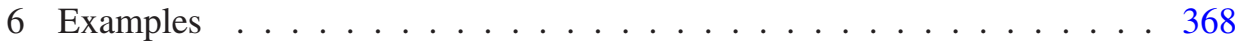

A Summary of notation and conventions . . . . . . . . . . 374

References . . . . . . . . . . . . . . . . . . . . . . . . . . 377 


\section{Introduction}

A fusion category $C$ is a semisimple, additive, rigid monoidal category. $\mathcal{C}$ is required to have only a finite number of simple objects up to isomorphism (we call this finitely semisimple) and to be $k$-linear over some field $k$ such that $\operatorname{Hom}(X, Y)$ is finitedimensional for all objects $X, Y \in|\zeta|$. In the following, we do not impose any condition on the field $k$, but we require that $\operatorname{End}(X) \cong k$ for all simple objects $X \in|\mathcal{C}|$ and say that $\mathcal{C}$ is split semisimple. For technical reasons, $\mathcal{C}$ is required to be essentially small, and for convenience, we equip every object $X \in|\mathcal{C}|$ with a specified left-dual. Such a rigid category is called left-autonomous. We do not require the monoidal unit $\mathbb{1}$ to be simple, i.e. we include the case of multi-fusion categories. For further background on fusion categories, we refer to [23], [1], and [4].

If a fusion category $\mathcal{C}$ arises as the category of comodules $\mathcal{M}^{H} \simeq \mathcal{C}$ of some Hopf algebra $H$,it admits a functor $F: \ell \rightarrow$ Vect $_{k}$ that is strong monoidal, i.e. in particular $F(X \otimes Y) \cong F X \otimes_{k} F Y$ are isomorphic vector spaces for all $X, Y \in|\ell|$. This happens because for every Hopf algebra $H$, the forgetful functor $\mathcal{M}^{H} \rightarrow$ Vect $_{k}$ is strong monoidal. The most interesting fusion categories are those that do not admit any strong monoidal functor to Vect $_{k}$ and therefore do not arise from Hopf algebras in this way.

Nevertheless, each fusion category $\mathcal{C}$ still admits the long canonical functor

$$
\begin{aligned}
\omega: \mathcal{C} \longrightarrow \operatorname{Vect}_{k}: X & \longrightarrow \operatorname{Hom}(\widehat{V}, \hat{V} \otimes X), \\
f & \left.\longmapsto \operatorname{(id}_{\widehat{V}} \otimes f\right) \circ-.
\end{aligned}
$$

Here, we have used the small progenerator

$$
\widehat{V}=\bigoplus_{j \in I} V_{j}
$$

where $\left\{V_{j}\right\}_{j \in I}$ is a set of representatives of the isomorphism classes of the simple objects of $\mathcal{C}$. The functor $\omega$ is $k$-linear, faithful, exact, and has a separable Frobenius structure, [6], [22], and [16], which includes the structure of both a lax and an oplax monoidal functor.

Under this functor, the $k$-dimension of a tensor product

$$
\operatorname{dim}_{k} \omega(X \otimes Y) \leq\left(\operatorname{dim}_{k} \omega X\right) \cdot\left(\operatorname{dim}_{k} \omega Y\right)
$$

is in general smaller than the product of $k$-dimensions. This effect is known as the truncation of the tensor product.

In the present article, we use the long canonical functor in order to arrive at a characterization of fusion categories in which we can fully parameterize the truncation of the tensor product in terms of combinatorial data. This is done as follows.

First, Tannaka-Kreĭn reconstruction has been generalized to functors with a separable Frobenius structure, [6], [15], [16], and [11]. It equips the universal coend 
$H=\operatorname{coend}(\mathcal{C}, \omega)$ with the structure of a weak Hopf algebra (WHA), [2] and [3]. This WHA can be shown to be finite-dimensional and split cosemisimple and to have commutative bases [16]. The long canonical functor plays the role of the forgetful functor $\mathcal{M}^{H} \rightarrow$ Vect $_{k}$ of the category $\mathcal{M}^{H}$ of finite-dimensional right $H$-comodules, and $\mathcal{C} \simeq \mathcal{M}^{H}$ are equivalent as $k$-linear additive monoidal categories.

We then choose an object $M \in|\mathcal{C}|$ that generates $\mathcal{C}$ as a fusion category, and define a finite directed graph $\mathcal{E}$, the dimension graph of $\mathcal{C}$ with respect to $M$. It depends on the choice of the generator $M$ and on the fusion coefficients of $\mathcal{C}$. The reason for considering this graph is the following.

The algebra $R=\operatorname{End}(\widehat{V}) \cong k^{|I|}$ has a basis of orthogonal idempotents $\lambda_{j}=$ $\operatorname{id}_{V_{j}}, j \in I$. The vector spaces $\omega X=\operatorname{Hom}(\hat{V}, \widehat{V} \otimes X)$ form $R$ - $R$-bimodules. We can choose a basis of $\omega X$ that consists of basis vectors of the $\operatorname{Hom}\left(V_{j}, V_{\ell} \otimes X\right)$ for all $j, \ell \in I$, i.e. one that is adapted to the orthogonal idempotents of $R$.

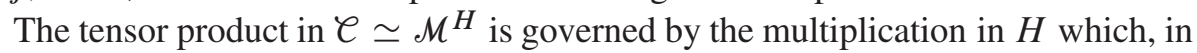
turn, is determined by the lax and oplax monoidal structure of $\omega$. The following is the lax monoidal structure:

$$
\begin{aligned}
\omega_{0}: k & \longrightarrow \omega \mathbb{1}, \\
1 & \longmapsto \rho_{\widehat{V}}^{-1},
\end{aligned}
$$

and

$$
\begin{aligned}
\omega_{X, Y}: \omega X \otimes \omega Y & \longrightarrow \omega(X \otimes Y), \\
f \otimes g & \longmapsto \alpha_{\hat{V}, X, Y} \circ\left(f \otimes \operatorname{id}_{Y}\right) \circ g .
\end{aligned}
$$

It is not difficult to see that $\omega(X \otimes Y) \cong \omega X \otimes_{R} \omega Y$ is the tensor product in the category of $R$-R-bimodules. In particular, if $f_{1} \in \operatorname{Hom}\left(V_{j}, V_{\ell} \otimes M\right)$ and $f_{2} \in$ $\operatorname{Hom}\left(V_{p}, V_{q} \otimes M\right), j, \ell, p, q \in I$, then $\omega_{M, M}\left(f_{1} \otimes f_{2}\right)$ is non-zero if and only if $q=j$.

We thus define the dimension graph $\mathscr{G}$ of $\mathcal{C}$ with respect to $M$ to have vertices $\mathscr{E}^{0}=I$, i.e. the orthogonal idempotents of $R$, and edges $\mathscr{E}_{\ell j}^{1}$ from $j$ to $\ell$ the vectors of a basis of $\operatorname{Hom}\left(V_{j}, V_{\ell} \otimes M\right)$. Then, two edges $f_{1}, f_{2} \in \mathscr{E}^{1}$ are composable if and only if the truncated tensor product $\omega(M \otimes M)$ contains the corresponding vector $f_{1} \otimes f_{2}$ of the $k$-linear tensor product $\omega M \otimes_{k} \omega M$.

There is a weak bialgebra (WBA) $H[\mathcal{E}]$ associated with the graph $\mathscr{E}$ and a surjection of WBAs $\pi: H[\mathscr{E}] \rightarrow H$ such that a specific simple comodule $k^{1} \mathscr{E}^{1}$ of $H[\mathscr{E}]$ is pushed forward under $\pi$ to the generating comodule $\omega M$ of $\mathcal{M}^{H}$. Since $\pi$ is a homomorphism of WBAs, the same holds for all tensor powers thereof, i.e. that $\left(k \mathscr{E}^{1}\right)^{\hat{\otimes} m}$ is pushed forward to $(\omega M)^{\hat{\otimes} m}, m \geq 0$.

In order to characterize $H$ and thereby the fusion category $\ell \simeq \mathcal{M}^{H}$, it remains to compute the kernel of $\pi$. This is done in two steps.

First, we take a suitable quotient $H[\mathscr{E}, \mathcal{E}]=H[\mathscr{E}] / I_{\mathscr{E}}$ in order to enforce that each $\left(k \mathscr{E}^{1}\right)^{\widehat{\otimes} m}, m \geq 0$, is equipped with the same endomorphism algebra as $(\omega M)^{\widehat{\otimes} m}$. 
This quotient is particularly easy if the monoidal unit $\mathbb{1}$ and the chosen generator $M$ of $\mathcal{C}$ are both simple and if $\mathcal{C}$ is braided such that braiding and inverse braiding of adjacent tensor factors already generate all endomorphisms of $(\omega M)^{\hat{\otimes} m}, m \geq 2$. In this case, the ideal $I_{\mathcal{E}}$ is generated by quadratic relations of the form ' $R T T=T T R$ ', a generalization of the construction of Faddeev-Reshetikhin-Takhtajan (FRT) [18] to WBAs. The coefficients of the $R$-matrix in these relations form the Boltzmann weight of a star-triangular face model.

Second, our surjection of WBAs factors through this first quotient yielding another surjection of WBAs $\bar{\pi}: H[\mathcal{E}, \mathcal{E}] \rightarrow H$. We show that ker $\bar{\pi}$ is generated by $1-g$ for a suitable subset of group-like elements $g \in H[\mathcal{E}, \mathcal{E}]$. Dividing by $1-g$ ensures that the categories of comodules of $H$ and $H[\mathcal{E}, \mathcal{E}] / \operatorname{ker} \pi$ agree everywhere, not just for fixed tensor powers of the generator $M$.

For the special case of $U_{q}\left(\mathfrak{s l}_{N}\right)$ in which the first quotient is given in terms of RTT relations, Hayashi [5] has already presented WHAs whose categories of finite-dimensional comodules have the same fusion rules as the modular categories associated with $U_{q}\left(\mathfrak{s l}_{N}\right)$. In fact, in this special case of our construction, the first quotient $H[\mathcal{E}] / I_{\mathscr{E}}$ appears in the literature on subfactors, see, for example [14]. In Ocneanu's terminology, the weak bialgebra $H[\mathcal{E}]$ is called a paragroup and the coefficients of the $R$-matrix a connection. The original FRT construction was reformulated by Müller [12] in a way that can be directly compared with our approach.

The present article is organized as follows. Section 2 summarizes some background material on WBAs and WHAs and on the generalization of Tannaka-Kre reconstruction to our case. In Section 3, we construct the dimension graph $\mathcal{E}$ and the surjection of WBAs $\pi: H[\mathscr{E}] \rightarrow H$. The first quotient $H[\mathscr{E}, \mathcal{E}]=H[\mathscr{E}] / I_{\mathscr{E}}$ is studied in Section 4. In Section 5, we study the group-like elements of the WBA $H[\mathcal{E}, \mathcal{E}]$ and their associated comodules in order to compute the kernel of $\bar{\pi}: H[\mathcal{E}, \mathcal{E}] \rightarrow H$. As examples, the modular categories associated with $U_{q}\left(\mathfrak{s l}_{2}\right)$ are treated in Section 6. The reader who is interested in a quick overview of our construction, is encouraged to go directly to that section. Appendix A contains a summary of the definitions and conventions for monoidal categories that we use.

Acknowledgements. The author is grateful to Gabriella Böhm, Catharina Stroppel, Kornél Szlachányi and Peter Vecsernyés for stimulating discussions, to Vladimir Turaev for correspondence, and to the anonymous referee for the hint that Theorem 5.6 holds for all homomorphisms between WBAs, a result which substantially simplified Section 5 .

\section{Preliminaries}

In Subsection 2.1, we summarize some key definitions and properties of weak bialgebras (WBAs) and weak Hopf algebras (WHAs) following [2] and [3]. Subsection 2.2 
reviews their categories of comodules following [16]. In Subsection 2.3, we recall the main results about the Tannaka-Kreĭn reconstruction of a WHA from a given monoidal category, [16] and [11].

We use the following notation. If $\mathcal{C}$ is a category, we write $X \in|\mathcal{C}|$ for the objects $X$ of $\mathcal{C}, \operatorname{Hom}(X, Y)$ for the collection of all morphisms $f: X \rightarrow Y$ and $\operatorname{End}(X)=\operatorname{Hom}(X, X)$. We denote the identity morphism of $X$ by $\operatorname{id}_{X}: X \rightarrow X$ and the composition of morphisms $f: X \rightarrow Y$ and $g: Y \rightarrow Z$ by $g \circ f: X \rightarrow Z$. If two objects $X, Y \in|\mathcal{C}|$ are isomorphic, we write $X \cong Y$. If two categories $\mathcal{C}$ and $\mathscr{D}$ are equivalent, we write $\mathcal{C} \simeq \mathscr{D}$. The identity functor on $\mathscr{C}$ is denoted by $1 \ell$. The category of vector spaces over a field $k$ is denoted by Vect $_{k}$ and its full subcategory of finite-dimensional vector spaces by fdVect $_{k}$. Both are $k$-linear, abelian and symmetric monoidal. The $n$-fold tensor power of some object $X \in|\mathcal{\ell}|$ of a monoidal category $(\mathcal{C}, \otimes, \mathbb{1}, \alpha, \lambda, \rho)$ is denoted by $X^{\otimes n}, n \in \mathbb{N}_{0}$. We set $X^{\otimes 0}=\mathbb{1}$. We use the notation $\mathbb{N}$ and $\mathbb{N}_{0}$ for the positive integers and the non-negative integers, respectively. For our notation and conventions regarding monoidal categories with duals as well as additive and abelian categories, we refer to Appendix A.

\subsection{Weak Hopf algebras}

Definition 2.1. A weak bialgebra $(H, \mu, \eta, \Delta, \varepsilon)$ over a field $k$ is a $k$-vector space $H$ such that

(1) $(H, \mu, \eta)$ is an associative algebra with multiplication $\mu: H \otimes H \rightarrow H$ and unit $\eta: k \rightarrow H$,

(2) $(H, \Delta, \varepsilon)$ is a coassociative coalgebra with comultiplication $\Delta: H \rightarrow H \otimes H$ and counit $\varepsilon: H \rightarrow k$,

(3) the following compatibility conditions hold:

$$
\begin{aligned}
\Delta \circ \mu & =(\mu \otimes \mu) \circ\left(\operatorname{id}_{H} \otimes \sigma_{H, H} \otimes \operatorname{id}_{H}\right) \circ(\Delta \otimes \Delta), \\
\varepsilon \circ \mu \circ\left(\mu \otimes \operatorname{id}_{H}\right) & =(\varepsilon \otimes \varepsilon) \circ(\mu \otimes \mu) \circ\left(\operatorname{id}_{H} \otimes \Delta \otimes \operatorname{id}_{H}\right) \\
& =(\varepsilon \otimes \varepsilon) \circ(\mu \otimes \mu) \circ\left(\operatorname{id}_{H} \otimes \Delta^{\circ p} \otimes \operatorname{id}_{H}\right), \\
\left(\Delta \otimes \operatorname{id}_{H}\right) \circ \Delta \circ \eta & =\left(\operatorname{id}_{H} \otimes \mu \otimes \operatorname{id}_{H}\right) \circ(\Delta \otimes \Delta) \circ(\eta \otimes \eta) \\
& =\left(\operatorname{id}_{H} \otimes \mu^{\mathrm{op}} \otimes \operatorname{id}_{H}\right) \circ(\Delta \otimes \Delta) \circ(\eta \otimes \eta) .
\end{aligned}
$$

Here $\sigma_{V, W}: V \otimes W \rightarrow W \otimes V, v \otimes w \mapsto w \otimes v$ is the transposition of the tensor factors, and by $\Delta^{\mathrm{op}}=\sigma_{H, H} \circ \Delta$ and $\mu^{\mathrm{op}}=\mu \circ \sigma_{H, H}$ we denote the opposite comultiplication and opposite multiplication, respectively. We tacitly identify the vector spaces $(V \otimes W) \otimes U \cong V \otimes(W \otimes U)$ and $V \otimes k \cong V \cong k \otimes V$, exploiting the coherence theorem for the monoidal category Vect $_{k}$.

A homomorphism $\varphi: H \rightarrow H^{\prime}$ of WBAs over the same field $k$ is a $k$-linear map that is a homomorphism of unital algebras as well as a homomorphism of counital coalgebras. 
In a WBA $H$, there are two important linear idempotents, the source counital map $\varepsilon_{s}=\left(\operatorname{id}_{H} \otimes \varepsilon\right) \circ\left(\operatorname{id}_{H} \otimes \mu\right) \circ\left(\sigma_{H, H} \otimes \mathrm{id}_{H}\right) \circ\left(\operatorname{id}_{H} \otimes \Delta\right) \circ\left(\operatorname{id}_{H} \otimes \eta\right): H \longrightarrow H$ and the target counital map $\varepsilon_{t}=\left(\varepsilon \otimes \mathrm{id}_{H}\right) \circ\left(\mu \otimes \mathrm{id}_{H}\right) \circ\left(\mathrm{id}_{H} \otimes \sigma_{H, H}\right) \circ\left(\Delta \otimes \mathrm{id}_{H}\right) \circ\left(\eta \otimes \mathrm{id}_{H}\right): H \longrightarrow H$. Their images $H_{s}=\varepsilon_{s}(H)$ and $H_{t}=\varepsilon_{t}(H)$ are mutually commuting unital subalgebras and are called the source base algebra and the target base algebra, respectively.

Definition 2.2. A weak Hopf algebra $(H, \mu, \eta, \Delta, \varepsilon, S)$ is a weak bialgebra $(H, \mu, \eta$, $\Delta, \varepsilon$ ) with a linear map $S: H \rightarrow H$ (antipode) that satisfies the following conditions:

$$
\begin{gathered}
\mu \circ\left(\operatorname{id}_{H} \otimes S\right) \circ \Delta=\varepsilon_{t}, \\
\mu \circ\left(S \otimes \operatorname{id}_{H}\right) \circ \Delta=\varepsilon_{s}, \\
\mu \circ\left(\mu \otimes \operatorname{id}_{H}\right) \circ\left(S \otimes \operatorname{id}_{H} \otimes S\right) \circ\left(\Delta \otimes \operatorname{id}_{H}\right) \circ \Delta=S .
\end{gathered}
$$

Note that if $f: H \rightarrow H^{\prime}$ is a homomorphism of WBAs and both $H$ and $H^{\prime}$ are WHAs, then $S^{\prime} \circ f=f \circ S$.

For convenience, we write $1=\eta(1)$ and omit parentheses in products, exploiting associativity. We also use Sweedler's notation and write $\Delta(x)=x^{\prime} \otimes x^{\prime \prime}$ for the comultiplication of $x \in H$ as an abbreviation of the expression $\Delta(x)=\sum_{k} a_{k} \otimes b_{k}$ with some $a_{k}, b_{k} \in H$. Similarly, we write $\left(\left(\Delta \otimes \operatorname{id}_{H}\right) \circ \Delta\right)(x)=x^{\prime} \otimes x^{\prime \prime} \otimes x^{\prime \prime \prime}$, exploiting coassociativity.

Definition 2.3. A coquasitriangular WHA $(H, \mu, \eta, \Delta, \varepsilon, S, r)$ over a field $k$ is a WHA $(H, \mu, \eta, \Delta, \varepsilon, S)$ over $k$ with a linear form $r: H \otimes H \rightarrow k$ (universal $r$-form) that satisfies the following conditions:

(1) for all $x, y \in H$,

$$
r(x \otimes y)=\varepsilon\left(x^{\prime} y^{\prime}\right) r\left(x^{\prime \prime} \otimes y^{\prime \prime}\right)=r\left(x^{\prime} \otimes y^{\prime}\right) \varepsilon\left(y^{\prime \prime} x^{\prime \prime}\right) ;
$$

(2) there exists a linear form $\bar{r}: H \otimes H \rightarrow k$ such that for all $x, y \in H$,

$$
\begin{aligned}
& \bar{r}\left(x^{\prime} \otimes y^{\prime}\right) r\left(x^{\prime \prime} \otimes y^{\prime \prime}\right)=\varepsilon(y x), \\
& r\left(x^{\prime} \otimes y^{\prime}\right) \bar{r}\left(x^{\prime \prime} \otimes y^{\prime \prime}\right)=\varepsilon(x y) ;
\end{aligned}
$$

(3) for all $x, y, z \in H$,

$$
\begin{aligned}
x^{\prime} y^{\prime} r\left(x^{\prime \prime} \otimes y^{\prime \prime}\right) & =r\left(x^{\prime} \otimes y^{\prime}\right) y^{\prime \prime} x^{\prime \prime}, \\
r((x y) \otimes z) & =r\left(y \otimes z^{\prime}\right) r\left(x \otimes z^{\prime \prime}\right), \\
r(x \otimes(y z)) & =r\left(x^{\prime} \otimes y\right) r\left(x^{\prime \prime} \otimes z\right) .
\end{aligned}
$$

The WHA $H$ is called cotriangular if in addition

$$
r\left(x^{\prime} \otimes y^{\prime}\right) r\left(y^{\prime \prime} \otimes x^{\prime \prime}\right)=\varepsilon(x y)
$$

for all $x, y \in H$. 
2.2. Comodules of weak Hopf algebras. We extend Sweedler's notation to the right $H$-comodules and write $\beta(v)=v_{0} \otimes v_{1}$ for the coaction $\beta: V \rightarrow V \otimes H$ of $H$ on some vector space $V$.

Proposition 2.4. Let $H$ be a WBA. Then the category $\mathcal{M}^{H}$ of finite-dimensional right $H$-comodules is a monoidal category $\left(\mathcal{M}^{H}, \hat{\otimes}, H_{s}, \alpha, \lambda, \rho\right)$. Here the monoidal unit object is the source base algebra $H_{s}$ with the coaction

$$
\begin{aligned}
\beta_{H_{s}}: H_{s} & \longrightarrow H_{s} \otimes H, \\
x & \longmapsto x^{\prime} \otimes x^{\prime \prime} .
\end{aligned}
$$

The tensor product $V \hat{\otimes} W=\operatorname{im} P_{V, W}$ of two right $H$-comodules $V, W \in\left|\mathcal{M}^{H}\right|$ is the truncated tensor product, which is the image of the $k$-linear idempotent

$$
\begin{aligned}
P_{V, W}: V \otimes W & \longrightarrow V \otimes W, \\
v \otimes w & \longmapsto\left(v_{0} \otimes w_{0}\right) \varepsilon\left(v_{1} w_{1}\right),
\end{aligned}
$$

with the coaction given by

$$
\begin{aligned}
\beta_{V \hat{\otimes} W}: V \hat{\otimes} W & \longrightarrow(V \hat{\otimes} W) \otimes H, \\
v \otimes w & \longmapsto\left(v_{0} \otimes w_{0}\right) \otimes\left(v_{1} w_{1}\right) .
\end{aligned}
$$

The unit constraints of the monoidal category are

$$
\begin{aligned}
\lambda_{V}: H_{s} \hat{\otimes} V & \longrightarrow V, \\
x \otimes v & \longmapsto v_{0} \varepsilon\left(x v_{1}\right),
\end{aligned}
$$

and

$$
\begin{aligned}
\rho_{V}: V \hat{\otimes} H_{s} & \longrightarrow V, \\
v \otimes x & \longmapsto v_{0} \varepsilon\left(v_{1} \varepsilon_{s}(x)\right),
\end{aligned}
$$

and the associator is inherited from that of $\mathbf{V e c t}_{k}$.

The forgetful functor of the category of finite-dimensional comodules of a WBA is not necessarily strong monoidal as in the case of a bialgebra, but it satisfies the following more general conditions of a functor with separable Frobenius structure [22].

Definition 2.5. Let $\mathcal{C}$ and $\ell^{\prime}$ be monoidal categories. A functor with Frobenius structure $\left(F, F_{X, Y}, F_{0}, F^{X, Y}, F^{0}\right): \mathcal{C} \rightarrow \ell^{\prime}$ is a functor $F: \mathcal{\ell} \rightarrow \ell^{\prime}$ that is lax monoidal as $\left(F, F_{X, Y}, F_{0}\right)$ and oplax monoidal as $\left(F, F^{X, Y}, F^{0}\right)$ and that satisfies the compatibility conditions

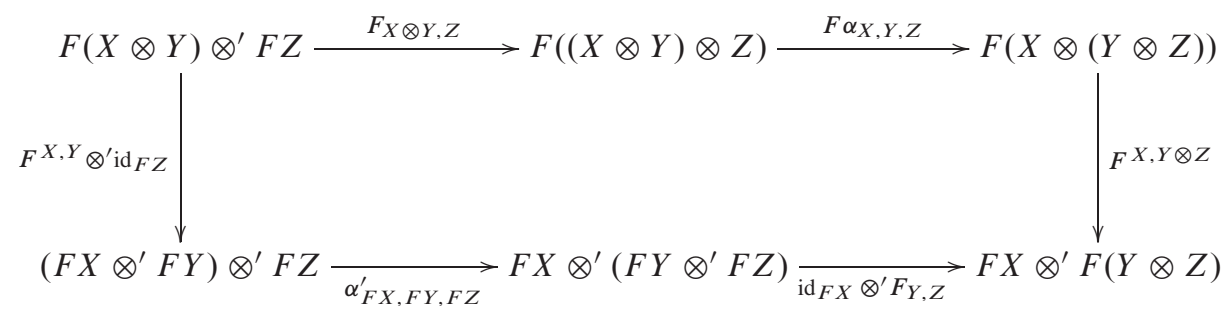


and

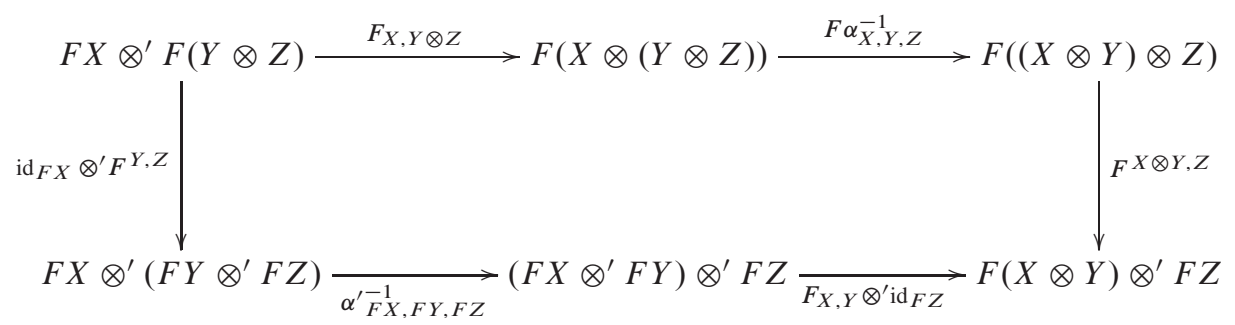

for all $X, Y, Z \in|\ell|$. It is called a functor with separable Frobenius structure if in addition

$$
F_{X, Y} \circ F^{X, Y}=\operatorname{id}_{F(X \otimes Y)},
$$

for all $X, Y \in|\ell|$.

This terminology was chosen because if $\boldsymbol{C}^{\prime}=$ Vect $_{k}$, the vector space $F \rrbracket$ forms a Frobenius algebra if $F$ has a Frobenius structure and an index-one Frobenius algebra if $F$ has a separable Frobenius structure, respectively. Frobenius algebras over a field are separable if and only if their Frobenius structure can be chosen to be of index one [9].

Proposition 2.6. Let $(H, \mu, \eta, \Delta, \varepsilon)$ be a WBA and $U: \mathcal{M}^{H} \rightarrow \operatorname{Vect}_{k}$ be the obvious forgetful functor. Then $\left(U, U_{X, Y}, U_{0}, U^{X, Y}, U^{0}\right)$ is a $k$-linear faithful functor with a separable Frobenius structure, and it takes values in $\mathbf{f d V e c t}_{k}$. The Frobenius structure is given by

$$
\begin{aligned}
U_{X, Y} & =\operatorname{coim} P_{X, Y}: U X \otimes U Y \longrightarrow P_{X, Y}(U X \otimes U Y), \\
U_{0} & =\eta: k \longrightarrow H_{S}, \\
U^{X, Y} & =\operatorname{im} P_{X, Y}: P_{X, Y}(U X \otimes U Y) \longrightarrow U X \otimes U Y, \\
U^{0} & =\left.\varepsilon\right|_{H_{S}}: H_{S} \longrightarrow k .
\end{aligned}
$$

Here $P_{X, Y}$ denotes the idempotent of (4) with its image factorization $P_{X, Y}=$ $\operatorname{im} P_{X, Y} \circ$ coim $P_{X, Y}$. Its image $P_{X, Y}(U X \otimes U Y)=U(X \hat{\otimes} Y)$ is the vector space underlying the truncated tensor product. Finally, $H_{S}=U \mathbb{1}$ is the vector space underlying the monoidal unit.

Proposition 2.7. Let $H$ be a WHA. Then $\mathcal{M}^{H}$ is left-autonomous if the left-dual of every object $V \in\left|\mathcal{M}^{H}\right|$ is chosen to be $\left(V^{*}, \mathrm{ev}_{V}, \operatorname{coev}_{V}\right)$, where the dual vector space $V^{*}$ is equipped with the coaction

$$
\begin{aligned}
\beta_{V^{*}}: V^{*} & \longrightarrow V^{*} \otimes H, \\
\vartheta & \longmapsto\left(v \mapsto \vartheta\left(v_{0}\right) \otimes S\left(v_{1}\right)\right),
\end{aligned}
$$


and the evaluation and coevaluation maps are given by

$$
\begin{aligned}
\mathrm{ev}_{V}: V^{*} \hat{\otimes} V & \longrightarrow H_{s}, \\
\vartheta \otimes v & \longmapsto \vartheta\left(v_{0}\right) \varepsilon_{s}\left(v_{1}\right),
\end{aligned}
$$

and

$$
\begin{aligned}
\operatorname{coev}_{V}: H_{s} & \longrightarrow V \hat{\otimes} V^{*} \\
x & \longmapsto \sum_{j}\left(\left(v_{j}\right)_{0} \otimes \vartheta^{j}\right) \varepsilon\left(x\left(v_{j}\right)_{1}\right) .
\end{aligned}
$$

Here we have used the evaluation and coevaluation maps that turn $V^{*}$ into a left-dual of $V$ in the category $\mathbf{~ f d V e c t}_{k}$ :

$$
\begin{aligned}
\mathrm{ev}_{V}^{\left(\text {fdVect }_{k}\right)}: V^{*} \otimes V & \longrightarrow k, \\
\vartheta \otimes v & \longmapsto \vartheta(v),
\end{aligned}
$$

and

$$
\begin{aligned}
\operatorname{coev}_{V}^{\left(\text {fdVect }_{k}\right)}: & k \longrightarrow V \otimes V^{*}, \\
1 & \longmapsto \sum_{j} v_{j} \otimes \vartheta^{j} .
\end{aligned}
$$

Let $V \in \mathcal{M}^{H}$ be a finite-dimensional right comodule of a WBA $H$ with some basis $\left\{e_{j}\right\}_{j}$. Then there are unique elements $c_{\ell j}^{(V)} \in H$ such that $\beta_{V}\left(e_{j}\right)=\sum_{\ell} e_{\ell} \otimes c_{\ell j}^{(V)}$ for all $j$. These $c_{\ell j}^{(V)}$ are called the coefficients of $V$ and their linear span the coefficient coalgebra $C(V) . C(V)$ is a subcoalgebra of $H$. If $H$ is a WHA, we call the element $t_{V}=\sum_{j} c_{j j}^{(V)} \in H$ the dual character of $V$.

\subsection{Tannaka-Kreĭn reconstruction}

Definition 2.8. Let $\mathcal{C}$ be an essentially small, finitely split semisimple, $k$-linear, additive monoidal category such that $k$ is a field and $\operatorname{Hom}(X, Y)$ is finite-dimensional over $k$ for all $X, Y \in \mathcal{C}$. By $\left\{V_{j}\right\}_{j \in I}$ where $I$ is a finite index set, we denote a set of representatives of the isomorphism classes of simple objects of $\mathcal{C}$. Then the long canonical functor is defined as

$$
\begin{aligned}
\omega: \mathcal{C} \rightarrow \operatorname{Vect}_{k}, \quad X & \mapsto \operatorname{Hom}(\widehat{V}, \widehat{V} \otimes X), \\
f & \left.\mapsto \operatorname{id}_{\widehat{V}} \otimes f\right) \circ-,
\end{aligned}
$$

where $\widehat{V}$ denotes the object

$$
\widehat{V}=\bigoplus_{j \in I} V_{j}
$$

Remark 2.9. The algebra $R=\operatorname{End}(\hat{V}) \cong \omega \mathbb{1} \cong k^{|I|}$ has a basis $\left(\lambda_{j}\right)_{j \in I}$ of orthogonal idempotents given by $\lambda_{j}=\mathrm{id}_{V_{j}} \in R$. It forms a Frobenius algebra $\left(R, \circ, \mathrm{id}_{R}, \Delta_{R}, \varepsilon_{R}\right)$ with comultiplication $\Delta_{R}: R \rightarrow R \otimes R$ and counit $\varepsilon_{R}: R \rightarrow k$ 
given by $\Delta\left(\lambda_{j}\right)=\lambda_{j} \otimes \lambda_{j}$ and $\varepsilon\left(\lambda_{j}\right)=1$ for all $j \in I$. The element $\Delta\left(\mathrm{id}_{R}\right)$ is a separability idempotent. Such a Frobenius algebra is called index one or Frobenius separable [9].

Proposition 2.10. Let $\mathcal{C}$ be as in Definition 2.8. Then the long canonical functor $\omega: \mathcal{C} \rightarrow$ Vect $_{k}$ is a $k$-linear faithful functor with a separable Frobenius structure $\left(\omega, \omega_{X Y}, \omega_{0}, \omega^{X Y}, \omega^{0}\right)$ and takes values in $\mathbf{f d V e c t}_{k}$. The separable Frobenius structure is given by

$$
\begin{aligned}
\omega_{0}: k & \longrightarrow \omega \mathbb{1}, \\
1 & \longmapsto \rho_{\hat{V}}^{-1}, \\
\omega_{X, Y}: \omega X \otimes \omega Y & \longrightarrow \omega(X \otimes Y), \\
f \otimes g & \longmapsto \alpha_{\hat{V}, X, Y} \circ\left(f \otimes \mathrm{id}_{Y}\right) \circ g, \\
\omega^{0}: \omega \mathbb{1} & \longrightarrow k, \\
v & \longmapsto \varepsilon_{R}\left(\rho_{\hat{V}} \circ v\right), \\
\omega^{X, Y}: \omega(X \otimes Y) & \longrightarrow \omega X \otimes \omega Y, \\
h & \longmapsto \sum_{j, \ell} \varepsilon_{R}\left(e_{(Y)}^{\ell} \circ\left(e_{(X)}^{j} \otimes \operatorname{id}_{Y}\right) \circ \alpha_{\hat{V}, X, Y}^{-1} \circ h\right) e_{j}^{(X)} \otimes e_{\ell}^{(Y)} .
\end{aligned}
$$

Here $\left(e_{j}^{(X)}\right)_{j}$ and $\left(e_{(X)}^{j}\right)_{j}$ denote a pair of dual bases of $\omega X=\operatorname{Hom}(\hat{V}, \hat{V} \otimes X)$ and $\operatorname{Hom}(\widehat{V} \otimes X, \widehat{V})$, respectively, with respect to the non-degenerate bilinear form

$$
g_{X}: \operatorname{Hom}(\hat{V} \otimes X, \hat{V}) \otimes \operatorname{Hom}(\hat{V}, \hat{V} \otimes X) \rightarrow k, \quad \vartheta \otimes v \mapsto \varepsilon_{R}(\vartheta \circ v) .
$$

Remark 2.11. (i) It can be shown that the long canonical functor already has a separable Frobenius structure if each simple object $X \in|\mathcal{C}|$ has $\operatorname{End}(X)$ a finitedimensional separable division algebra over $k$. Such an algebra admits an index one Frobenius structure [9]. For our construction below in terms of the dimension graph, however, we require the stronger condition that $\operatorname{End}(X) \cong k$.

(ii) Since $\mathcal{C}$ is semisimple, there is no need to worry about exactness of $\omega$ at this point. Thanks to the equivalence $\mathcal{C} \simeq \mathcal{M}^{H}$ in Theorem 2.12 below (see [16]), $\mathcal{C}$ is abelian and $\omega$ exact.

By a generalization of Tannaka-Kreר̆n reconstruction from strong monoidal functors to functors with separable Frobenius structure, we obtain the following characterization of $\mathcal{C}$ as the category $\ell \simeq \mathcal{M}^{H}$ of finite-dimensional comodules over the universal coend $H=\operatorname{coend}(\mathcal{C}, \omega)$. The long canonical functor appears as the forgetful functor $\omega: \mathcal{M}^{H} \rightarrow$ Vect $_{k}$. 
Theorem 2.12. Let $\mathcal{C}$ be as in Definition 2.8. Then $\mathcal{C} \simeq \mathcal{M}^{H}$ are equivalent as $k$-linear, additive monoidal categories. Here $H=\operatorname{coend}(\mathcal{C}, \omega)$ is a finitedimensional split cosemisimple WBA such that $H_{s} \cong R \cong H_{t}$. The WBA $H$ is a direct sum of matrix coalgebras,

$$
H=\bigoplus_{j \in I}\left(\omega V_{j}\right)^{*} \otimes \omega V_{j},
$$

with operations

$$
\begin{aligned}
\mu\left([\vartheta \mid v]_{X} \otimes[\zeta \mid w]_{Y}\right) & =\left[\zeta \circ\left(\vartheta \otimes \operatorname{id}_{Y}\right) \circ \alpha_{\widehat{V}, X, Y}^{-1} \mid \alpha_{\hat{V}, X, Y} \circ\left(v \otimes \operatorname{id}_{Y}\right) \circ w\right]_{X \otimes Y}, \\
\eta(1) & =\left[\rho_{\widehat{V}} \mid \rho_{\widehat{V}}^{-1}\right]_{\mathbb{\mathbb { N }}}, \\
\Delta\left([\vartheta \mid v]_{X}\right) & =\sum_{j}\left[\vartheta \mid e_{j}^{(X)}\right]_{X} \otimes\left[e_{(X)}^{j} \mid v\right]_{X}, \\
\varepsilon\left([\vartheta \mid v]_{X}\right) & =\varepsilon_{R}(\vartheta \circ v) .
\end{aligned}
$$

Here we write $[\vartheta \mid v]_{X} \in(\omega X)^{*} \otimes \omega X$ with $v \in \omega X, \vartheta \in \operatorname{Hom}(\hat{V} \otimes X, \widehat{V}) \cong(\omega X)^{*}$ and simple $X \in|\mathcal{C}|$ for the homogeneous elements of $H$. The precise form of the universal coend as a colimit also allows us to use the same expression for arbitrary objects of $\mathcal{C}$, but subject to the relations that $[\zeta \mid(\omega f)(v)]_{Y}=\left[(\omega f)^{*}(\zeta) \mid v\right]_{X}$ for all $v \in \omega X, \zeta \in(\omega Y)^{*}$ and for all morphisms $f: X \rightarrow Y$ of $\mathcal{C}$. Recall that $(\omega f)(v)=\left(\operatorname{id}_{\hat{V}} \otimes f\right) \circ v$ and $(\omega f)^{*}(\zeta)=\zeta \circ\left(\operatorname{id}_{\hat{V}} \otimes f\right)$.

If in addition, $\mathcal{C}$ is left-autonomous, then $H$ forms a WHA with antipode

$$
S\left(\left[e_{(X)}^{j} \mid e_{\ell}^{(X)}\right]_{X}\right)=\left[\tilde{e}_{\left(X^{*}\right)}^{\ell} \mid \tilde{e}_{j}^{\left(X^{*}\right)}\right]_{X^{*}}
$$

where $\left(\tilde{e}_{j}^{\left(X^{*}\right)}\right)_{j}$ denotes the basis of $\omega\left(X^{*}\right)$ defined by

$$
\tilde{e}_{j}^{\left(X^{*}\right)}=\left(e_{(X)}^{j} \otimes \operatorname{id}_{X^{*}}\right) \circ \alpha_{\widehat{V}, X, X^{*}}^{-1} \circ\left(\operatorname{id}_{\widehat{V}} \otimes \operatorname{coev}_{X}\right) \circ \rho_{\widehat{V}}^{-1},
$$

and where $\left(\tilde{e}_{\left(X^{*}\right)}^{j}\right)_{j}$ is the basis dual to it with respect to the bilinear form $g_{X^{*}}, c f$. (5).

Remark 2.13. (i) If the monoidal unit $\mathbb{\square}$ is simple, the base algebras intersect trivially, $H_{s} \cap H_{t} \cong k$. If $\mathcal{C}$ is braided, $H$ is coquasi-triangular and $\mathscr{C} \simeq \mathcal{M}^{H}$ an equivalence of braided monoidal categories. If $\mathcal{C}$ is symmetric monoidal, $H$ is cotriangular. Further structure and properties of $\mathcal{C}$ such as a pivotal structure, a ribbon structure, or the properties that a pivotal category $\ell$ be spherical or that a ribbon category $\mathcal{C}$ be modular, can be translated into additional structure and properties of $H=$ $\operatorname{coend}(\mathcal{C}, \omega)$ as well, [16] and [17].

(ii) Note that if $X \in|\mathcal{}|$ is an arbitrary object, then $\omega X$ forms a right- $H$ comodule with the coaction

$$
\begin{aligned}
\beta_{\omega X}: \omega X & \longrightarrow \omega X \otimes H \\
v & \longmapsto \sum_{j} e_{j}^{(X)} \otimes\left[e_{(X)}^{j} \mid v\right]_{X} .
\end{aligned}
$$


Its coefficient coalgebra is given by $C(X)=\left((\omega X)^{*} \otimes \omega X\right) / N_{X} \subseteq H$ where the subspace $N_{X} \subseteq(\omega X)^{*} \otimes \omega X$ is generated by the elements

$$
[\vartheta \mid(\omega f)(v)]_{X}-\left[(\omega f)^{*}(\vartheta) \mid v\right]_{X}
$$

for all $v \in \omega X, \vartheta \in(\omega X)^{*}$ and $f \in \operatorname{End}(X)$.

\section{A combinatorial cover of the universal coend}

In order to develop a combinatorial description of a given category $\ell$ with the properties as in Definition 2.8, we first construct a WBA $H[\mathscr{E}]$ in combinatorial terms and a surjection $\pi: H[\mathscr{E}] \rightarrow H$ onto the universal coend $H=\operatorname{coend}(\mathcal{C}, \omega)$.

3.1. Weak bialgebras associated with finite directed graphs. Let $\mathscr{E}=\left(\mathscr{E}^{0}, \mathscr{E}^{1}\right)$ be a finite directed graph with a set $\mathscr{E}^{0}$ of vertices and a set $\mathscr{\ell}^{1} \subseteq \mathscr{E}^{0} \times \mathscr{E}^{0}$ of edges. We use the following notation and terminology. Every edge $p=\left(v_{0}, v_{1}\right) \in \mathscr{g}^{1}$ has a source and a target vertex, denoted by $\sigma(p)=v_{1} \in \mathscr{G}^{0}$ and $\tau(p)=v_{0} \in \mathscr{E}^{0}$, respectively. We also set $\sigma(v)=v=\tau(v)$ for all $v \in \mathscr{E}^{0}$. By

$$
\mathscr{E}^{m}=\left\{\left(p_{1}, \ldots, p_{m}\right) \in\left(\mathscr{E}^{1}\right)^{m} \mid \sigma\left(p_{j}\right)=\tau\left(p_{j+1}\right) \text { for all } 1 \leq j \leq m-1\right\},
$$

we denote the set of paths of length $m$ in $\mathscr{G}, m \in \mathbb{N}$. Finally, for vertices $v, w \in \mathscr{E}^{0}$, the set

$$
\mathscr{E}_{w v}^{m}=\left\{p \in \mathcal{E}^{m} \mid \sigma(p)=v, \tau(p)=w\right\}
$$

contains all paths of length $m \in \mathbb{N}_{0}$ from $v$ to $w$.

We write $p q \in \mathscr{Q}^{\ell+m}$ for the concatenation of two paths $p \in \mathscr{\ell}^{\ell}$ and $q \in \mathscr{\ell}^{m}$ provided that $\sigma(p)=\tau(q)$. The free $k$-vector space on the set $\mathscr{E}^{m}$ is denoted by $k \mathscr{E}^{m}, m \in \mathbb{N}_{0}$.

Proposition 3.1. Let $\mathscr{E}$ be a finite directed graph. There is a WBA $(H[\mathscr{E}], \mu, \eta, \Delta, \varepsilon)$ with the underlying vector space

$$
H[\mathscr{E}]=\coprod_{m \in \mathbb{N}_{0}}\left(k \mathscr{E}^{m}\right)^{*} \otimes k \mathscr{E}^{m}
$$

and operations

$$
\begin{aligned}
\eta(1) & =\sum_{j, \ell \in \mathscr{S}^{0}}[j \mid \ell]_{0}, \\
\mu\left([p \mid q]_{m} \otimes[r \mid s]_{\ell}\right) & =\delta_{\sigma(p), \tau(r)} \delta_{\sigma(q), \tau(s)}[p r \mid q s]_{m+\ell}, \\
\Delta\left([p \mid q]_{m}\right) & =\sum_{r \in \mathscr{S}^{m}}[p \mid r]_{m} \otimes[r \mid q]_{m},
\end{aligned}
$$




$$
\varepsilon\left([p \mid q]_{m}\right)=\delta_{p q},
$$

for all $p, q \in \mathcal{G}^{m}, r, s \in \mathscr{G}^{\ell}, m, \ell \in \mathbb{N}_{0}$. Here, Ц is the coproduct in Vect $_{k}$, and we have denoted basis vectors of the homogeneous components $H[\mathcal{E}]_{m}=$ $\left(k \mathscr{E}^{m}\right)^{*} \otimes k^{\mathscr{E}^{m}}$ of $H[\mathscr{E}]$ by $[p \mid q]_{m}=p \otimes q \in\left(k \mathscr{E}^{m}\right)^{*} \otimes k^{\mathscr{G}^{m}}$. As usual, we write $\delta_{p q}=1$ if $p=q$ and $\delta_{p q}=0$ if $p \neq q$ for all $p, q \in \mathcal{G}^{m}, m \in \mathbb{N}_{0}$.

Proof. Direct verification.

Proposition 3.2. Let $\mathscr{E}$ be a finite directed graph and $H[\mathcal{E}]$ as in Proposition 3.1.

(1) The source and target counital maps of $H[\mathscr{E}]$ are given by

$$
\begin{aligned}
& \varepsilon_{S}\left([p \mid q]_{m}\right)=\delta_{p, q} \sum_{j \in \mathscr{E}^{0}}[j \mid \sigma(p)]_{0}, \\
& \varepsilon_{t}\left([p \mid q]_{m}\right)=\delta_{p, q} \sum_{j \in \mathscr{E}^{0}}[\tau(q) \mid j]_{0},
\end{aligned}
$$

for all $p, q \in \mathscr{G}^{m}, m \in \mathbb{N}_{0}$.

(2) $H[\mathcal{E}]$ is split cosemisimple. Its simple right comodules are the vector spaces $k \mathscr{G}^{m}, m \in \mathbb{N}_{0}$, with the coactions

$$
\begin{aligned}
\beta_{k \mathscr{G}^{m}: k \mathscr{E}^{m}} & \longrightarrow k \mathscr{E}^{m} \otimes H[\mathscr{E}], \\
p & \longmapsto \sum_{q \in \mathscr{E}^{m}} q \otimes[q \mid p]_{m} .
\end{aligned}
$$

(3) The truncated tensor product of $\mathcal{M}^{H[\mathcal{E}]}$ is such that

$$
k \mathscr{E}^{m} \hat{\otimes} k \mathscr{E}^{\ell} \cong k \mathscr{E}^{m+\ell}
$$

for all $m, \ell \in \mathbb{N}_{0}$.

(4) The unital algebra underlying $H[\mathcal{E}]$ is graded with homogeneous components $H[\mathscr{E}]_{m}$ of degree $m \in \mathbb{N}_{0}$.

(5) As an associative algebra or as a unital associative algebra, $H[\mathcal{E}]$ is generated by the set $H[\mathscr{G}]_{0} \cup H[\mathscr{E}]_{1}$.

Proof. Part (1) is established by a direct computation. Part (2) holds because the homogeneous components $H\left[\mathscr{E}_{m}\right.$ are matrix coalgebras with coefficients in $k$. For Part (3), we compute the idempotent (4) and find that for all $m, \ell \in \mathbb{N}_{0}, p \in \mathcal{E}^{m}$, $q \in \mathscr{E}^{\ell}$,

$$
P_{k \mathscr{E}^{m}, k \mathscr{G}^{\ell}}(p \otimes q)= \begin{cases}p \otimes q & \text { if } \sigma(p)=\tau(q), \\ 0 & \text { otherwise. }\end{cases}
$$

Part (4) holds because multiplication in $H[\mathcal{E}]$ is zero unless the paths in both components of $[-\mid-]$ are composable. Part (5) holds because the length of paths is additive under concatenation. 
Remark 3.3. (i) The algebra underlying $H[\mathscr{E}]$ is the path algebra $k \Gamma$ of the quiver $\Gamma=\mathscr{E} \times \mathscr{E}$, up to identifying $\left(\mathrm{k}^{m}\right)^{*}=\mathrm{k}^{\mathrm{m}}$. We do not use the terminology quiver in the present article because it is not the category of modules over $k \Gamma$, but rather that of comodules that is related to our fusion category $\ell$.

(ii) The category $\mathcal{M}^{H[\mathscr{E}]}$ of finite-dimensional right- $H[\mathcal{E}]$ comodules is an essentially small, split semisimple, $k$-linear, abelian monoidal category whose isomorphism classes of simple objects are indexed by non-negative integers $m \in \mathbb{N}_{0}$. The tensor product is given by $m \otimes \ell \cong m+\ell$ for all $m, \ell \in \mathbb{N}_{0}$. The forgetful functor $U: \mathcal{M}^{H[\mathscr{E}]} \rightarrow$ Vect $_{k}$ is such that $U m \cong k \mathscr{E}^{m}$. Conversely, $H[\mathscr{E}] \cong$ $\operatorname{coend}\left(\mathcal{M}^{H[\varepsilon]}, U\right)$.

3.2. The fundamental surjection. Although the truncation of the tensor product in Proposition 3.2(3) is rather elementary, this is the mechanism that controls the truncation of the tensor product in all fusion categories. We demonstrate this by constructing a surjection $H[\mathscr{E}] \rightarrow H$.

Definition 3.4. Let $\mathcal{C}$ be an essentially small, finitely split semisimple, $k$-linear, additive left-autonomous monoidal category such that $k$ is a field and $\operatorname{Hom}(X, Y)$ is finite-dimensional over $k$ for all $X, Y \in|\mathcal{E}|$. An object $M \in|\mathcal{E}|$ is said to generate $\mathcal{C}$ as a fusion category if the following conditions are satisfied.

(1) Every simple object $V_{j}, j \in I$, of $\mathcal{C}$ appears as a direct summand of $M^{\otimes n}$ for some $n \in \mathbb{N}_{0}$.

(2) The object $M$ is multiplicity free, i.e. if $M \cong X \oplus Y \oplus Z$, then $X \not Y$.

(3) The monoidal unit $\mathbb{1}$ and $M$ have pairwise non-isomorphic direct summands, i.e. if $\mathbb{1} \cong X \oplus Y$ and $M \cong Z \oplus W$, then $X \nsubseteq Z$.

Remark 3.5. (i) Part (1) is the usual definition, but (2) and (3) can be required in addition without loss of generality. Note that Part (3) rules out the trivial fusion category, but every non-trivial such category does have a generating object.

(ii) The monoidal unit $\mathbb{1}$ is always multiplicity-free [4]. In the present section and in Section 4, the assumption that $\mathcal{C}$ be autonomous can be dropped if one requires instead that the monoidal unit be multiplicity-free.

Given a fusion category $\ell$ with a generating object $M \in|\ell|$, we now choose a graph $\mathcal{E}$ in such a way that we obtain a surjection of WBAs $\pi: H[\mathscr{E}] \rightarrow H$. Then the composability of paths in $\mathscr{E}$ which controls the multiplication in $H[\mathcal{E}]$, also governs the truncated tensor product in $\mathcal{C} \cong \mathcal{M}^{H}$.

Definition 3.6. Let $\mathcal{C}$ be an essentially small, finitely split semisimple, $k$-linear, additive, left-autonomous monoidal category such that $k$ is a field and $\operatorname{Hom}(X, Y)$ is finite-dimensional over $k$ for all $X, Y \in|\mathcal{C}|$. Let $M \in|\mathcal{C}|$ be an object that generates $\mathcal{C}$ and let $\left\{V_{j}\right\}_{j \in I}$ denote a set of representatives of the isomorphism classes of simple 
objects of $\mathcal{C}$. The dimension graph $\mathcal{E}$ of $\mathcal{C}$ with respect to $M$ is the finite directed graph whose set of vertices is $\mathscr{E}^{0}=I$ and whose set $\mathscr{E}_{\ell j}^{1}$ of edges from $j \in \mathscr{E}^{0}$ to $\ell \in \mathscr{E}^{0}$ is a basis of $\operatorname{Hom}\left(V_{j}, V_{\ell} \otimes M\right)$.

Remark 3.7. If $M \in|\mathcal{C}|$ is simple and, say, $M \cong V_{1}$, then the adjacency matrix of $\mathcal{G}$ is the fusion matrix $N_{1}$ with coefficients $\left(N_{1}\right)_{j \ell}=\operatorname{dim}_{k} \operatorname{Hom}\left(V_{j}, V_{\ell} \otimes V_{1}\right)$.

We denote a basis of $\omega M=\operatorname{Hom}(\hat{V}, \widehat{V} \otimes M)$ by $\left\{e_{p}^{(M)}\right\}_{p}$ and by $\left\{e_{(M)}^{q}\right\}_{q}$ its dual basis with respect to the bilinear form $g_{M}$ of (5). We also choose the basis $\left\{e_{j}^{(\mathbb{1})}\right\}_{j}$, $e_{j}^{(\mathbb{1})}=\rho_{\hat{V}}^{-1} \circ \lambda_{j}$ of $\omega \mathbb{1}=\operatorname{Hom}(\hat{V}, \hat{V} \otimes \mathbb{1})$ whose dual basis with respect to $g_{\mathbb{1}}$ is given by $\left\{e_{(\mathbb{1})}^{\ell}\right\}_{\ell}$ with $e_{(\mathbb{1})}^{\ell}=\lambda_{\ell} \circ \rho_{\hat{V}}$. Observe that $k \mathscr{E}^{1}=\omega M$ and $k \mathscr{E}^{0} \cong \omega \mathbb{1}$ which we identify in the following.

Theorem 3.8. Let $\mathcal{C}$ be as in Definition 3.6, $M \in|\mathcal{C}|$ be an object that generates $\mathcal{C}$ and $\mathcal{E}$ be the dimension graph of $\mathcal{C}$ with respect to $M$. We denote by $H=\operatorname{coend}(\mathcal{C}, \omega)$ the universal coend with respect to the long canonical functor, $c f$. Theorem 2.12. Then there is a surjection of WBAs $\pi: H[\mathcal{E}] \rightarrow H$ as follows.

(1) $\pi\left([j \mid \ell]_{0}\right)=\left[e_{(\mathbb{1})}^{j} \mid e_{\ell}^{(\mathbb{1})}\right]_{\mathbb{1}}$ for all $j, \ell \in \mathcal{E}^{0}$.

(2) $\pi\left([p \mid q]_{1}\right)=\left[e_{(M)}^{p} \mid e_{q}^{(M)}\right]_{M}$ for all $p, q \in \mathscr{E}^{1}$.

(3) $\pi$ pushes forward the right $H[\mathscr{E}]$-comodule $k^{\mathscr{E}^{1}}$ to the right $H$-comodule $\omega M$, i.e. $\left(\operatorname{id}_{k \mathscr{E} 1} \otimes \pi\right) \circ \beta_{k \mathscr{E} 1}=\beta_{\omega M}$.

(4) $\pi$ also pushes forward the right $H[\mathcal{E}]$-comodule $k \mathscr{E}^{0}$ to the right $H$-comodule $\omega \mathbb{1}$, i.e. $\left(\mathrm{id}_{k} \mathscr{0} 0 \pi\right) \circ \beta_{k} \mathscr{0}=\beta_{\omega \mathbb{1}}$.

Proof. As an associative algebra, $H[\mathscr{G}]$ is generated by $H[\mathscr{G}]_{0} \cup H[\mathscr{G}]_{1}$, i.e. Parts (1) and (2) fix the value of $\pi$ on a set of generators of $H[\mathscr{g}]$.

Given that $\mathcal{E}$ is the dimension graph, (1) and (2) are compatible with the multiplication of $H[\mathcal{E}]$. This can be seen by computing all products of degree- 0 and degree- 1 terms of $H[\mathscr{E}]$ and their images under $\pi$. Therefore, Parts (1) and (2) define a unique linear map $\pi: H[\mathscr{E}] \rightarrow H$ which forms a homomorphism of associative algebras. This map $\pi$ is also compatible with the units as can be seen by inspection.

In order to see that $\pi$ respects the comultiplication, we show in a direct computation that $\Delta(\pi(a))=(\pi \otimes \pi)(\Delta(a))$ for all generators $a \in H[\mathscr{G}]_{0} \cup H[\mathscr{E}]_{1}$. Then, by induction, if this claim holds for some $a, b \in H[\mathscr{E}]$, we also have

$$
\begin{aligned}
\Delta(\pi(a b)) & =\Delta(\pi(a) \pi(b)) \\
& =\left(\pi(a)^{\prime} \pi(b)^{\prime}\right) \otimes\left(\pi(a)^{\prime \prime} \pi(b)^{\prime \prime}\right) \\
& =\left(\pi\left(a^{\prime}\right) \pi\left(b^{\prime}\right)\right) \otimes\left(\pi\left(a^{\prime \prime}\right) \pi\left(b^{\prime \prime}\right)\right) \\
& =\pi\left(a^{\prime} b^{\prime}\right) \otimes \pi\left(a^{\prime \prime} b^{\prime \prime}\right)
\end{aligned}
$$




$$
=(\pi \otimes \pi)(\Delta(a b)),
$$

because $\pi$ respects the multiplication, because $H$ is a WBA; because of the assumption, because $\pi$ respects the multiplication, and because $H[\mathscr{E}]$ is a WBA.

The map $\pi$ also respects the counit. On generators $a \in H[\mathscr{E}]_{0} \cup H[\mathscr{E}]_{1}$, we see by inspection that $\varepsilon(\pi(a))=\varepsilon(a)$. Then, by induction, if this claim holds for some $a, b \in H[\mathcal{E}]$, we find that

$$
\begin{aligned}
\varepsilon(\pi(a b)) & =\varepsilon(\pi(a) \pi(b)) \\
& =\varepsilon(\pi(a) 1 \pi(b)) \\
& =\varepsilon\left(\pi(a) 1^{\prime}\right) \varepsilon\left(1^{\prime \prime} \pi(b)\right) \\
& =\varepsilon\left(\pi(a) \pi(1)^{\prime}\right) \varepsilon\left(\pi(1)^{\prime \prime} \pi(b)\right) \\
& =\varepsilon\left(\pi(a) \pi\left(1^{\prime}\right)\right) \varepsilon\left(\pi\left(1^{\prime \prime}\right) \pi(b)\right) \\
& =\varepsilon\left(\pi\left(a 1^{\prime}\right)\right) \varepsilon\left(\pi\left(1^{\prime \prime} b\right)\right) \\
& =\varepsilon(a b),
\end{aligned}
$$

because $\pi$ respects the multiplication; $H$ is a WBA; $\pi$ respects the unit; $\pi$ respects the comultiplication, and $\pi$ respects the multiplication. The last equality of (6) is shown by a direct computation for generic $a=[p \mid q]_{m} \in H[\mathcal{E}], b=[r \mid s]_{\ell} \in H[\mathcal{E}]$.

At this point, we know that $\pi$ is a homomorphism of WBAs. It is surjective because $\mathcal{C}$ is generated by $M$ and already the image $\pi\left(H[\mathcal{G}]_{1}\right)$ exhausts the coefficient coalgebra $C(M)=(\omega M)^{*} \otimes \omega M$.

Parts (3) and (4) can finally be seen in a direct computation.

Remark 3.9. If both the monoidal unit $\mathbb{1}$ and the generating object $M$ are simple, then the restriction $\left.\pi\right|_{H[\mathscr{E}]_{0} \oplus H[\mathscr{E}]_{1}}: H[\mathscr{E}]_{0} \oplus H[\mathscr{E}]_{1} \rightarrow H$ is injective.

\section{Schur-Weyl dual description at fixed powers of $M$}

In this section, we define a quotient of the WBA $H[\mathcal{E}]$ in such a way that the tensor powers of the generating object $M$ have the desired endomorphism algebras.

\subsection{Implementing the endomorphisms of the tensor powers of $M$}

Definition 4.1. Let $\zeta$ and $M$ be as in Theorem 3.8. An endomorphism system for $\bigodot$ with respect to $M$ is a sequence $\mathcal{E}=\left(E^{(n)}\right)_{n \in \mathbb{N}_{0}}$ of sets $E^{(n)} \subseteq \operatorname{End}\left(M^{\otimes n}\right)$ of endomorphisms such that

(1) End( $\mathbb{1})$ as an associative algebra is generated by $E^{(0)} \cup\left\{\operatorname{id}_{\mathbb{1}}\right\}$;

(2) for all $n \in \mathbb{N}$, $\operatorname{End}\left(M^{\otimes n}\right)$ is generated by

$$
\left(E^{(n-1)} \otimes \operatorname{id}_{M}\right) \cup E^{(n)},
$$


where we have abbreviated

$$
E^{(n-1)} \otimes \operatorname{id}_{M}=\left\{f^{(n-1)} \otimes \operatorname{id}_{M} \mid f^{(n-1)} \in E^{(n-1)}\right\} .
$$

The situation is particularly easy if both $\mathbb{1}$ and $M$ are simple and if $\mathcal{C}$ is braided with braiding $\psi_{X, Y}: X \otimes Y \rightarrow Y \otimes X$ such that the endomorphism algebras are already generated by the braiding and inverse braiding of adjacent tensor factors. For $n \geq 2$, we denote by $B_{n}$ the associative unital algebra generated by $\left\{\psi_{j}^{ \pm} \mid 1 \leq j \leq n-1\right\}$ with

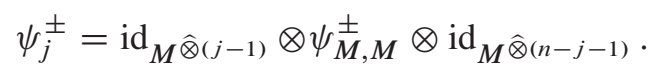

Definition 4.2. Let $\mathcal{C}$ and $M$ be as in Theorem 3.8. Then $\mathcal{C}$ is said to satisfy the strong Schur-Weyl property if both $\mathbb{1}$ and $M$ are simple and if $\mathcal{C}$ is braided such that $\operatorname{End}\left(M^{\otimes n}\right)=B_{n}$ for all $n \geq 2$.

Example 4.3. Let $\mathcal{C}$ and $M$ be as in Theorem 3.8 and assume that $\mathcal{C}$ satisfies the strong Schur-Weyl property. Then an endomorphism system for $\mathcal{C}$ with respect to $M$ is given by

$$
\begin{aligned}
& E^{(1)}=\emptyset, \\
& E^{(0)}=\emptyset \text {, } \\
& E^{(2)}=\left\{\psi_{M, M}, \psi_{M, M}^{-1}\right\},
\end{aligned}
$$

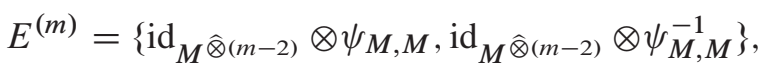

for all $m \geq 3$.

Given an endomorphism system $\mathcal{E}=\left(E^{(n)}\right)_{n \in \mathbb{N}_{0}}$ for $\mathcal{C}$ with respect to $M$, we can express the endomorphisms $\omega f^{(n)}:(\omega M)^{\widehat{\otimes} n} \rightarrow(\omega M)^{\widehat{\otimes} n}, f^{(n)} \in E^{(n)}, n \in \mathbb{N}$, as

$$
\left(\omega f^{(n)}\right)\left(e_{p_{1}}^{(M)} \otimes \cdots \otimes e_{p_{n}}^{(M)}\right)=\sum_{r_{1}, \ldots, r_{n} \in \mathcal{G}^{1}} e_{r_{1}}^{(M)} \otimes \cdots \otimes e_{r_{n}}^{(M)} f_{r_{1} \cdots r_{n} ; p_{1} \cdots p_{n}}^{(n)},
$$

with coefficients $f_{r_{1} \cdots r_{n} ; p_{1} \cdots p_{n}}^{(n)} \in k$. By analogy, for $n=0$ and $\omega f^{(0)}: \omega \mathbb{1} \rightarrow \omega \mathbb{1}$, $f^{(0)} \in E^{(0)}$, this is replaced by

$$
\left(\omega f^{(0)}\right)\left(e_{j}^{(\mathbb{1})}\right)=\sum_{\ell \in \mathscr{S}^{0}} e_{\ell}^{(\mathbb{1})} f_{\ell ; j}^{(0)},
$$

with coefficients $f_{\ell ; j}^{(0)} \in k$.

Remark 4.4. (i) Recall that $\operatorname{End}\left(M^{\otimes n}\right) \cong \operatorname{End}\left((\omega M)^{\hat{\otimes} n}\right)$ in view of the equivalence $\mathcal{C} \simeq \mathcal{M}^{H}$ of $k$-linear monoidal categories. 
(ii) Because of the form of the long canonical functor, $(\omega f)(v)=\left(\mathrm{id}_{\widehat{V}} \otimes f\right) \circ v$ for $v \in \omega X, f: X \rightarrow Y, X, Y \in|\ell|$, and so the coefficients $f_{r_{1} \cdots r_{n} ; p_{1} \cdots p_{n}}^{(n)}$ in (4.1) are zero unless $\tau\left(r_{1}\right)=\tau\left(p_{1}\right)$ and $\sigma\left(r_{n}\right)=\sigma\left(p_{n}\right)$.

Definition 4.5. Let $\mathcal{C}, M$ and $\mathcal{G}$ be as in Theorem 3.8 and $\mathcal{E}=\left(E^{(n)}\right)_{n \in \mathbb{N}_{0}}$ be an endomorphism system for $\mathcal{C}$ with respect to $M$. The endomorphism adapted WBA is the quotient $H[\mathcal{E}, \mathcal{E}]=H[\mathcal{E}] / I_{\mathcal{E}}$ where $I_{\mathscr{E}}$ is the two-sided ideal generated by the relations

$$
\begin{aligned}
\sum_{p_{1}, \ldots, p_{n} \in \mathcal{G}^{1}}\left[r_{1} \mid p_{1}\right]_{1} \cdots\left[r_{n} \mid p_{n}\right]_{1} f_{p_{1} \cdots p_{n} ; q_{1} \cdots q_{n}}^{(n)} & \\
& -\sum_{p_{1}, \ldots, p_{n} \in \mathcal{E}^{1}} f_{r_{1} \cdots r_{n} ; p_{1} \cdots p_{n}}^{(n)}\left[p_{1} \mid q_{1}\right]_{1} \cdots \cdot\left[p_{n} \mid q_{n}\right]_{1}
\end{aligned}
$$

for all $r_{j}, q_{j} \in \mathcal{G}^{1}, j \in\{1, \ldots, n\}, f^{(n)} \in E^{(n)}$ and $n \in \mathbb{N}_{0}$.

Note that all relations in the quotient $H[\mathcal{E}] / I_{\mathcal{E}}$ are homogeneous, and so $H[\mathcal{E}, \mathcal{E}]$ is graded, cf. Proposition 3.2(4).

Proposition 4.6. The endomorphism adapted $W B A H[\mathcal{E}, \mathcal{E}]$ of Definition 4.5 forms $a W B A$.

Proof. We have to show that $I_{\mathcal{E}}$ is also a two-sided coideal, i.e. it satisfies $\Delta\left(I_{\mathcal{E}}\right) \subseteq$ $I_{\mathcal{E}} \otimes H+H \otimes I_{\mathcal{E}}$ and $I_{\mathcal{E}} \subseteq \operatorname{ker} \varepsilon$. This is established in a direct computation. Note that the relations that generate the ideal $I_{\mathcal{E}}$, can be rewritten as

$$
\begin{aligned}
& \sum_{\substack{p_{1}, \ldots, p_{n} \in \mathcal{E}^{1}: \\
\sigma\left(p_{j}\right)=\tau\left(p_{j+1}\right)}}\left[r_{1} \cdots r_{n} \mid p_{1} \cdots p_{n}\right]_{n} f_{p_{1} \cdots p_{n} ; q_{1} \cdots q_{n}}^{(n)} \\
& \quad-\sum_{\substack{p_{1}, \ldots, p_{n} \in \mathcal{E}^{1}: \\
f_{1} \cdots r_{n} ; p_{1} \cdots p_{n} \\
\sigma\left(p_{j}\right)=\tau\left(p_{j+1}\right)}}^{\left.f_{1}^{(n)} \cdots p_{n} \mid q_{1} \cdots q_{n}\right]_{n} .}
\end{aligned}
$$

Proposition 4.7. Under the assumptions of Definition 4.5, the surjection $\pi: H[\mathcal{E}] \rightarrow$ $H$ of Theorem 3.8 factors through the canonical projection $H[\mathcal{E}] \rightarrow H[\mathcal{E}, \mathcal{E}]$, giving rise to another surjection of WBAs $\bar{\pi}: H[\mathcal{E}, \mathcal{E}] \rightarrow H$. This map $\bar{\pi}$ also satisfies the properties (1) to (4) of Theorem 3.8 and in addition

(5) The restriction $\left.\bar{\pi}\right|_{H[\mathcal{E}, \mathcal{E}]_{0} \oplus H[\mathcal{E}, \mathcal{E}]_{1}}: H[\mathcal{G}, \mathcal{E}]_{0} \oplus H[\mathcal{E}, \mathcal{E}]_{1} \rightarrow H$ is injective. Proof. In any quotient of $H[\mathcal{E}]$, the relation (7) holds for a particular $f^{(n)} \in E^{(n)}$, $n \in \mathbb{N}_{0}$, if and only if the linear map

$$
\begin{aligned}
\left(k^{\mathcal{G}^{1}}\right)^{\hat{\otimes} n} & \longrightarrow\left(k^{\mathcal{G}^{1}}\right)^{\hat{\otimes} n}, \\
p_{1} \otimes \cdots \otimes p_{n} & \longmapsto \sum_{r_{1}, \ldots, r_{n} \in \mathcal{G}^{1}} r_{1} \otimes \cdots \otimes r_{n} f_{r_{1} \cdots r_{n} ; p_{1} \cdots p_{n}}^{(n)}
\end{aligned}
$$


forms a morphism of right comodules. This is established in a direct calculation. The claim holds because $\pi$ pushes forward $k \mathscr{E}^{0}$ to $\omega \mathbb{1}$ and $k \mathscr{E}^{1}$ to $\omega M$, and because $\pi$ is a morphism of WBAs and therefore preserves tensor products. For Property (5), we recall that $M$ satisfies all conditions of Definition 3.4 and that the direct summands of $\mathbb{1}$ are pairwise non-isomorphic [4].

The special case of the endomorphism system of Example 4.3 is particularly interesting because in this case, the ideal $I_{\mathcal{E}}$ is generated by quadratic relations only.

Definition 4.8. Let $\mathcal{C}, M$ and $\mathcal{E}$ be as in Theorem 3.8 and the endomorphism system $\mathscr{E}=\left(E^{(n)}\right)_{n \in \mathbb{Z}_{\geq 0}}$ be as in Example 4.3. We denote the coefficients of the braiding under the long canonical functor by $R_{r_{1} r_{2} ; p_{1} p_{2}}$, i.e.

$$
\omega\left(\psi_{M, M}\right)\left(p_{1} \otimes p_{2}\right)=\sum_{r_{1}, r_{2} \in \mathcal{G}^{1}} r_{1} \otimes r_{2} R_{r_{1} r_{2} ; p_{1} p_{2}},
$$

for all $p_{1}, p_{2} \in \mathcal{E}^{1}$. The weak Faddeev-Reshetikhin-Takhtajan (FRT) bialgebra is the quotient $H[\mathscr{E}, R]=H[\mathscr{E}] / I_{R}$ where $I_{R}$ is the two-sided ideal generated by the relations

$$
\begin{aligned}
\sum_{p_{1}, p_{2} \in \mathcal{E}^{1}}\left[r_{1} \mid p_{1}\right]_{1} \cdot\left[r_{2} \mid p_{2}\right]_{1} R_{p_{1} p_{2} ; q_{1} q_{2}} & \\
& -\sum_{p_{1}, p_{2} \in \mathcal{E}^{1}} R_{r_{1} r_{2} ; p_{1} p_{2}}\left[p_{1} \mid q_{1}\right]_{1} \cdot\left[p_{2} \mid q_{2}\right]_{1},
\end{aligned}
$$

for all $r_{1}, r_{2}, q_{1}, q_{2} \in \mathcal{E}^{1}$.

Note that the relations can again be written in a slightly different fashion:

$$
\sum_{\substack{p_{1}, p_{2} \in \mathcal{E}^{1}: \\ \sigma\left(p_{1}\right)=\tau\left(p_{2}\right)}}\left[r_{1} r_{2} \mid p_{1} p_{2}\right]_{2} R_{p_{1} p_{2} ; q_{1} q_{2}}-\sum_{\substack{p_{1}, p_{2} \in \mathcal{E}^{1}: \\ \sigma\left(p_{1}\right)=\tau\left(p_{2}\right)}} R_{r_{1} r_{2} ; p_{1} p_{2}}\left[p_{1} p_{2} \mid q_{1} q_{2}\right]_{2} .
$$

In complete analogy to Proposition 4.6, the weak FRT bialgebra $H[\mathcal{G}, R]$ forms a WBA. We call it the weak FRT bialgebra because our quotient generalizes the construction of Faddeev-Reshetikhin-Takhtajan [18] to finite-dimensional split cosemisimple WBAs. This is the situation in which Hayashi describes WHAs whose categories of finite-dimensional comodules have the same fusion rules as the modular categories associated with $U_{q}\left(\mathfrak{s l}_{N}\right)$ at suitable roots of unity [5]. Since $R$ consists of the coefficients of the braiding, $R$ satisfies a generalization of the quantum Yang-Baxter equation to truncated tensor products. Such an $R$-matrix is known in the physics literature as the Boltzmann weight of a star-triangular face model. $R$-matrices of this type were studied, for example, in [7]. 
Example 4.9. If $\mathcal{C}$ and its endomorphism system $\mathcal{E}=\left(E^{(n)}\right)_{n \in \mathbb{N}_{0}}$ are as in Example 4.3, the endomorphism adapted WBA coincides with the weak FRT bialgebra.

Proof. We have to show that the quadratic relations that define $I_{R}$ in (8) already generate the entire two-sided ideal $I_{\mathcal{E}}$ of Definition 4.5. Recall that $E^{(0)}=\emptyset=E^{(1)}$ in this example. First, since $R$ is the braiding, each relation (8) for $R$ implies another relation of the same form with $R^{-1}$ rather than $R$. Just multiply the relation (8) with $R^{-1}$ from the right and from the left. Second, in order to show that all elements (7) of degree greater than two are already contained in the two-sided ideal generated by $I_{R}$, it is sufficient to verify that all endomorphisms of the form $\omega f=\mathrm{id}_{(\omega M)^{\hat{\otimes}(n-2)}} \otimes \omega\left(\sigma_{M, M}^{ \pm}\right)$, $f \in E^{(n)}, n \geq 3$, are already implemented by the quotient modulo $I_{R}$. This is done in a direct computation.

Even in situations in which $\zeta$ is braided and the braiding and its inverse do not generate all endomorphisms of the tensor powers of $\omega M$, the weak FRT bialgebra is worth studying in more detail. Firstly, the weak FRT bialgebra is equipped with a coquasi-triangular structure and, secondly, the endomorphism adapted WBA is a quotient of the weak FRT bialgebra.

Proposition 4.10. Let $\mathcal{C}, M$ and $\mathcal{G}$ be as in Theorem 3.8 and assume in addition that $\mathcal{C}$ is braided. Then the WBA $H[\mathcal{E}, R]$ of Definition 4.8 is coquasi-triangular with universal $r$-form $r: H[\mathcal{G}, R] \otimes H[\mathcal{E}, R] \rightarrow k$ given by

$$
\begin{aligned}
& r\left([u \mid v]_{0} \otimes[w \mid x]_{0}\right)=\delta_{u, v} \delta_{v, x} \delta_{u, w}, \\
& r\left([u \mid v]_{0} \otimes[p \mid q]_{1}\right)=\delta_{u, \tau(p)} \delta_{v, \sigma(p)} \delta_{p, q}, \\
& r\left([p \mid q]_{0} \otimes[u \mid v]_{0}\right)=\delta_{\sigma(p), u} \delta_{\tau(p), v} \delta_{p, q}, \\
& r\left([p \mid q]_{1} \otimes[r \mid s]_{1}\right)=\left\{\begin{array}{cc}
R_{p r, s q} & \text { if } \tau(p)=\tau(s), \sigma(r)=\sigma(q), \\
0 & \sigma(s)=\tau(q), \sigma(p)=\tau(r), \\
0 & \text { otherwise, }
\end{array}\right.
\end{aligned}
$$

and further, inductively, by

$$
r\left([p \mid q]_{m} \otimes[r \mid s]_{\ell+1}\right)=\sum_{t \in \mathscr{G}^{m}} r\left([p \mid t]_{m} \otimes\left[r_{1} \mid s_{1}\right]_{\ell}\right) r\left([t \mid q]_{m} \otimes\left[r_{2} \mid s_{2}\right]_{1}\right),
$$

where $r=r_{1} r_{2}, s=s_{1} s_{2}$ with $r_{1}, s_{1} \in \mathscr{G}^{\ell}$ and $r_{2}, s_{2} \in \mathscr{G}^{1}$; and by

$$
r\left([p \mid q]_{m+1} \otimes[r \mid s]_{\ell}\right)=\sum_{t \in \mathscr{G} \ell} r\left(\left[p_{2} \mid q_{2}\right]_{1} \otimes[r \mid t]_{\ell}\right) r\left(\left[p_{1} \mid q_{1}\right]_{m} \otimes[t \mid s]_{\ell}\right),
$$

where $p=p_{1} p_{2}$ and $q=q_{1} q_{2}$ with $p_{1}, q_{1} \in \mathcal{E}^{m}$ and $p_{2}, q_{2} \in \mathcal{E}^{1}$. The map $\pi_{R}=\bar{\pi} \circ \pi^{\prime}: H[\mathcal{G}, \mathcal{R}] \rightarrow H$ is a surjective homomorphism of coquasi-triangular WBAs. 
Proof. Direct computation.

Corollary 4.11. Let $\mathcal{C}, M$, and $\mathcal{E}$ be as in Theorem 3.8 and assume in addition that $\mathcal{C}$ is braided. Then there is a surjection of coquasi-triangular WBAs $\pi^{\prime}: H[\mathcal{E}, R] \rightarrow$ $H[\mathcal{G}, \mathcal{E}]$ and the Boltzmann weight

$$
\begin{aligned}
R: k \mathscr{E}^{1} \hat{\otimes} k \mathscr{E}^{1} & \longrightarrow k \mathscr{E}^{1} \hat{\otimes} k \mathscr{E}^{1}, \\
p_{1} \otimes p_{2} & \longmapsto \sum_{r_{1}, r_{2} \in \mathscr{E}^{1}} r_{1} \otimes r_{2} R_{r_{1} r_{2} ; p_{1} p_{2}},
\end{aligned}
$$

is star-triangular, i.e.

$$
R_{1} \circ R_{2} \circ R_{1}=R_{2} \circ R_{1} \circ R_{2},
$$

where $R_{1}=R \otimes \mathrm{id}_{\omega M}$ and $R_{2}=\mathrm{id}_{\omega M} \otimes R$.

Proof. Since for all $n \geq 2, B_{n} \leq \operatorname{End}\left(M^{\otimes n}\right)$ always forms a subalgebra, we have $I_{R} \subseteq I_{\mathcal{E}}$. A compatible coquasi-triangular structure on $H[\mathcal{E}, \mathcal{E}]$ always exists because the braiding is, of course, a morphism. The Boltzmann weight is star triangular because $R$ coincides with the coefficients of the braiding $\omega\left(\psi_{M, M}\right): \omega M \hat{\otimes} \omega M \rightarrow$ $\omega M \hat{\otimes} \omega M$.

4.2. Comparing the categories of comodules. In this section, we compare the categories $\mathcal{M}^{H[\mathscr{G}, \mathcal{E}]}$ and $\mathcal{M}^{H}$ of finite-dimensional comodules of the endomorphism adapted WBA $H[\mathcal{E}, \mathcal{E}]$ and of the universal coend $H=\operatorname{coend}(\mathcal{C}, \omega)$.

Proposition 4.12. Let $\mathcal{C}, M$ and $\mathcal{G}$ be as in Theorem 3.8 and $\mathcal{E}=\left(E^{(n)}\right)_{n \in \mathbb{N}_{0}}$ be an endomorphism system for $C$ with respect to $M$.

(1) The vector space $(\omega M)^{\hat{\otimes} n}, n \in \mathbb{N}_{0}$, is both an $H[\mathcal{E}, \mathcal{E}]$ - and an $H$-comodule. The homomorphism of WBAs $\bar{\pi}: H[\mathcal{E}, \mathcal{E}] \rightarrow H$ pushes forward the $H[\mathcal{E}, \mathcal{E}]$-comodule structure to the $H$-comodule structure, i.e.

$$
\left.\operatorname{id}_{(\omega M)^{\hat{\otimes} n}} \otimes \bar{\pi}\right) \circ \beta_{(\omega M)^{\otimes} n}^{(H[\mathcal{E}, \mathcal{E}])}=\beta_{(\omega M)^{\otimes} n}^{(H)} .
$$

(2) A linear map $f:(\omega M)^{\widehat{\otimes} n} \rightarrow(\omega M)^{\hat{\otimes} n}, n \in \mathbb{N}_{0}$, is $H$-colinear if and only if it is $H[\mathcal{G}, \mathcal{E}]$-colinear, i.e.

$$
\operatorname{End}_{\mathcal{M}^{H}[\mathcal{S}, \mathcal{E}]}\left((\omega M)^{\hat{\otimes} n}\right)=\operatorname{End}_{\mathcal{M}^{H}}\left((\omega M)^{\hat{\otimes} n}\right) .
$$

(3) A linear subspace $V \subseteq(\omega M)^{\widehat{\otimes} n}, n \in \mathbb{N}_{0}$, is an $H[\mathcal{E}, \mathcal{E}]$-subcomodule if and only if it is an $H$-subcomodule.

(4) Let $V \subseteq(\omega M)^{\hat{\otimes} n}$ and $W \subseteq(\omega M)^{\hat{\otimes} m}, n, m \in \mathbb{N}_{0}$, be right $H[\mathcal{E}, \mathcal{E}]$-subcomodules. If $n \neq m$, then $V \nsubseteq W$ are not isomorphic as $H[\mathcal{E}, \mathcal{E}]$-comodules. If $n=m$, then $V$ and $W$ are isomorphic as $H[\mathcal{G}, \mathcal{E}]$-comodules if and only if they are isomorphic as $H$-comodules. 
(5) $H[\mathcal{E}, \mathcal{E}]$ is split cosemisimple. The isomorphism classes of its simple comodules can be represented by some $V_{j}^{(m)} \in\left|\mathcal{M}^{H[\mathcal{G}, \mathcal{E}]}\right|$ where $m \in \mathbb{N}_{0}$ and $j \in$ $\left\{1, \ldots, \ell_{m}\right\}, \ell_{m} \in \mathbb{N}$. Here, $j$ labels the isomorphism classes of simple $H$-comodules in the complete decomposition of $(\omega M)^{\hat{\otimes} m}$ as an $H$-comodule. Furthermore, $\bar{\pi}$ pushes forward each $V_{j}^{(m)}$ to that particular isomorphism type of $\mathrm{H}$-comodules.

Proof. (1) Theorem 3.8, Parts (3) and (4) and the fact that $\bar{\pi}$ is a homomorphism of algebras.

(2) If $f$ is $H[\mathscr{E}, \mathscr{E}]$-colinear, then it is also $H$-colinear, using the homomorphism of coalgebras $\bar{\pi}: H[\mathscr{G}, \mathcal{E}] \rightarrow H$. Conversely, if $f$ is $H$-colinear, then $f$ is contained in the $k$-linear span of the set of all finite products of elements of the form $\omega f^{(m)} \otimes$ id $_{(\omega M)^{\hat{\otimes}(n-m)}}, 0 \leq m \leq n, f^{(m)} \in E^{(m)}$. Each endomorphism of the spanning set is right $H[\mathscr{E}, \mathscr{E}]$-colinear by Proposition 4.7 , and so $f$ is $H[\mathscr{E}, \mathcal{E}]$-colinear as well.

(3) Let $V$ be an $H[\mathscr{E}, \mathcal{E}]$-subcomodule, i.e. we have $\beta_{(\omega M)^{\otimes}}^{(H[\mathscr{G}, \mathscr{E}])}(V) \subseteq V \otimes H[\mathscr{E}, \mathscr{E}]$. Applying $\left(\mathrm{id}_{(\omega M)} \hat{\otimes}^{\hat{\otimes}_{n}} \otimes \bar{\pi}\right)$ shows that $\beta_{(\omega M)}^{(H)}{ }_{(\omega n}^{\otimes \otimes}(V) \subseteq V \otimes H$. Conversely, let $V$ be an $H$-subcomodule. Since $H$ is cosemisimple, $(\omega M)^{\hat{\otimes} n}=V \oplus W$ with some subcomodule $W$. The linear map $f:(\omega M)^{\hat{\otimes} n} \rightarrow(\omega M)^{\hat{\otimes} n}$ with $\left.f\right|_{V}=\operatorname{id}_{V}$ and $\left.f\right|_{W}=0$ forms a morphism of $H$-comodules. By Part (2), $f$ is $H[\mathscr{E}, \mathscr{E}]$-colinear and therefore its image $V$ an $H[\mathscr{G}, \mathcal{E}]$-subcomodule.

(4) If $n \neq m$, the coefficient coalgebras $C(V) \subseteq H[\mathcal{E}, \mathcal{E}]_{n}$ and $C(W) \subseteq$ $H[\mathcal{E}, \mathcal{E}]_{m}$ are in different degree, and so $C(V) \cap C(W)=\{0\}$ which implies the claim. In the case in which $n=m$, we use Part (2).

(5) In order to show that $H[\mathscr{E}, \mathcal{E}]$ is split cosemisimple, we show that it is a coproduct (direct sum) of matrix coalgebras.

First, since the ideal $I_{\mathcal{E}}$ in Definition 4.5 is generated by homogeneous elements, the canonical projection $p: H[\mathcal{E}] \rightarrow H[\mathcal{E}, \mathcal{E}]=H[\mathcal{E}] / I_{\mathcal{E}}$ preserves the grading of the algebra. Therefore,

$$
H[\mathcal{E}, \mathcal{E}]=\coprod_{m \in \mathbb{N}_{0}} H[\mathcal{E}, \mathcal{E}]_{m} .
$$

Here, the homogeneous components $H[\mathscr{G}, \mathscr{E}]_{m}=p\left(\left(k \mathscr{E}^{m}\right)^{*} \otimes k \mathscr{E}^{m}\right)$ form the coefficient coalgebras $H[\mathscr{E}, \mathcal{E}]_{m}=C\left((\omega M)^{\widehat{\otimes} m}\right)$. Recall that $p$ pushes forward the $H[\mathscr{E}]$-comodule $k^{\mathscr{E}^{m}}$ to the $H[\mathscr{E}, \mathcal{E}]$-comodule $(\omega M)^{\hat{\otimes} m}$ (Theorem 3.8(3) and Part (1)).

Since $H$ is split cosemisimple, $(\omega M)^{\hat{\otimes} m}$ as an $H$-comodule decomposes into

$$
(\omega M)^{\hat{\otimes} m} \cong \underbrace{V_{1}^{(m)} \oplus \cdots \oplus V_{1}^{(m)}}_{p_{1}} \oplus \cdots \oplus \underbrace{V_{\ell_{m}}^{(m)} \oplus \cdots \oplus V_{\ell_{m}}^{(m)}}_{p_{\ell_{m}}}, \quad \ell_{m} \in \mathbb{N} .
$$


Here, the $V_{j}^{(m)}, 1 \leq j \leq \ell_{m}$ are pairwise non-isomorphic $H$-comodules, and the $p_{j} \in \mathbb{N}$ denote their multiplicities. Furthermore, $\operatorname{End}_{\mathcal{M}^{H}}\left(V_{j}^{(m)}\right) \cong k$ for all $j$.

By Part (3), each instance of a $V_{j}^{(m)} \subseteq(\omega M)^{\widehat{\otimes} m}$ forms an $H[\mathcal{E}, \mathcal{E}]$-subcomodule, and by Part (2), $\operatorname{End}_{\mathcal{M} H[\varepsilon, \mathcal{E}]}\left(V_{j}^{(m)}\right) \cong k$, and so (12) is a complete decomposition of $H[\mathcal{G}, \mathcal{E}]$-comodules as well.

If we write $V=(\omega M)^{\hat{\otimes} m}$ and $E=\operatorname{End}_{\mathcal{M} H[\mathcal{E}, \mathcal{E}]}(V)$, the construction of $H[\mathscr{E}, \mathcal{E}]$ in Definitions 4.1 and 4.5 shows that

$$
H[\mathcal{E}, \mathcal{E}]_{m}=V^{*} \otimes_{E} V .
$$

Thanks to (12), $E$ is known to be the product of matrix algebras

$$
E=\bigoplus_{j=1}^{\ell_{m}} k^{p_{j} \times p_{j}},
$$

and a direct computation shows that

$$
H[\mathcal{E}, \mathcal{E}]_{m}=V^{*} \otimes_{E} V=\bigoplus_{j=1}^{\ell_{m}}\left(V_{j}^{(m)}\right)^{*} \otimes V_{j}^{(m)} .
$$

Combining this with (11) proves the claim.

\section{Comparing different powers of $M$}

In order to fully understand the relationship between the categories of comodules of $H[\mathcal{E}, \mathcal{E}]$ and of $H$, we determine the preimage under $\hat{\pi}$ (Proposition 4.12(5)) of the simple comodules of $H$, i.e. all those simple $H[\mathscr{E}, \mathcal{E}]$-comodules that are pushed forward by $\bar{\pi}$ to the same simple $H$-comodule.

In the following, the assumption that $\ell$ be left-autonomous, i.e. that $H=$ $\operatorname{coend}(\mathcal{C}, \omega)$ is a WHA, is not only used to imply that the monoidal unit is multiplicityfree, but is rather a key to comparing the categories of comodules of $H[\mathcal{G}, \mathcal{E}]$ and of $H$.

The assumption guarantees that it is sufficient to determine the preimage under $\hat{\pi}$ of the monoidal unit $\omega \mathbb{1}$. It turns out that all simple isomorphism types in that preimage are obtained by conjugating the monoidal unit of $\mathcal{M}^{H[\mathcal{E}, \mathcal{E}]}$ by group-like elements $g$ such that $\bar{\pi}(g)=1$. Dividing the endomorphism adapted WBA $H[\mathcal{E}, \mathcal{E}]$ by $1-g$ for these group-likes $g$ then yields a WHA that is isomorphic to $H$.

5.1. Group-like comodules. In this subsection, we consider an arbitrary WBA $H$.

Definition 5.1. An element $g \in H$ of a WBA $H$ is called 
(1) right group-like if $\Delta g=g 1^{\prime} \otimes g 1^{\prime \prime}$ and $\varepsilon_{S}(g)=1$,

(2) left group-like if $\Delta g=1^{\prime} g \otimes 1^{\prime \prime} g$ and $\varepsilon_{t}(g)=1$,

(3) group-like if it is both right and left group-like.

The set of group-like elements in a WBA forms a monoid. Note that we do not require the group-like elements of a WBA to have a multiplicative inverse. If $H$ is a WHA, however, every group-like $g \in H$ has the inverse $g^{-1}=S(g)$.

Proposition 5.2. Let $H$ be a WBA.

(1) For each group-like $g \in H$, there is a right $H$-comodule structure on the vector space $H_{s}$ given by

$$
\begin{aligned}
\beta_{H_{S}^{g}}: H_{s} & \longrightarrow H_{s} \otimes H, \\
x & \longmapsto x_{0} \otimes\left(g x_{1}\right),
\end{aligned}
$$

where $\beta_{\mathbb{1}}: H_{S} \rightarrow H_{s} \otimes H, x \mapsto x_{0} \otimes x_{1}$ denotes the right $H$-comodule structure on $H_{s}$ that yields the monoidal unit of $\mathcal{M}^{H}$, cf. (3). In the following, we denote the comodule with the structure (13) by $H_{s}^{g}$.

(2) If $g_{1}, g_{2} \in H$ are group-like, then

$$
H_{s}^{g_{1}} \widehat{\otimes} H_{s}^{g_{2}} \cong H_{s}^{g_{1} g_{2}}
$$

Proof. Parts (1) and (3) are straightforward. For part (2), the left-unit constraint of

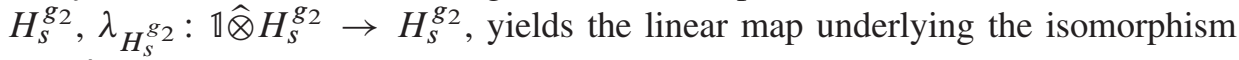
$H_{S}^{g_{1}} \hat{\otimes} H_{S}^{g_{2}} \rightarrow H_{S}^{g_{1} g_{2}}$.

Given a homomorphism of bialgebras $f: H \rightarrow H^{\prime}$ and the induced push-forward functor $f^{*}: \mathcal{M}^{H} \rightarrow \mathcal{M}^{H^{\prime}}$, the $H$-comodules sent by $f^{*}$ to the monoidal unit $\mathbb{1} \in$ $\left|\mathcal{M}^{H^{\prime}}\right|$ are precisely the comodules $H_{S}^{g}$ for the group-like elements $g \in H$ that satisfy $f(g)=1$. This is established in the remainder of this subsection and will be applied to the homomorphism $\bar{\pi}: H[\mathcal{E}, \mathcal{E}] \rightarrow H$.

Proposition 5.3 (from [20], Lemma 4.1). In every WBA $H$, the restriction

$$
\left.\varepsilon_{t}\right|_{H_{s}}: H_{s} \longrightarrow H_{t}
$$

forms an algebra anti-isomorphism with inverse $\left.\bar{\varepsilon}_{s}\right|_{H_{t}}$ where $\bar{\varepsilon}_{S}(x)=1^{\prime} \varepsilon\left(1^{\prime \prime} x\right)$ for all $x \in H$.

Proposition 5.4 (from [20, Lemma 6.3]). Let $f: H \rightarrow H^{\prime}$ be a homomorphism of WBAs. Then the restrictions $\left.f\right|_{H_{s}}: H_{s} \rightarrow H_{s}^{\prime}$ and $\left.f\right|_{H_{t}}: H_{t} \rightarrow H_{t}^{\prime}$ form isomorphisms of algebras. Their inverses are $\left.f\right|_{H_{S}} ^{-1}(y)=1^{\prime} \varepsilon\left(f\left(1^{\prime \prime}\right) y\right)$ for all $y \in H_{S}^{\prime}$ and $\left.f\right|_{H_{t}}{ }^{-1}(z)=\varepsilon\left(z f\left(1^{\prime}\right)\right) 1^{\prime \prime}$ for all $z \in H_{t}^{\prime}$, respectively. 
The following proposition generalizes some results of Nikshych [13] and Vecsernyés [24] from WHAs to WBAs.

Proposition 5.5. Let $H$ be a WBA. (1) There is a one-to-one correspondence between right group-like elements $g \in H$ and right $H$-coactions $\beta: H_{s} \rightarrow H_{s} \otimes H$ that satisfy

$$
\left(\operatorname{id}_{H_{S}} \otimes \varepsilon_{S}\right) \circ \beta=\left(\operatorname{id}_{H_{S}} \otimes \varepsilon_{S}\right) \circ \Delta \circ \varepsilon_{S} .
$$

The correspondence is given by $g=\varepsilon\left(1_{0}\right) 1_{1}$ and $\beta(x)=\varepsilon_{s}\left(x^{\prime}\right) \otimes g x^{\prime \prime}$ for all $x \in H_{s}$.

(2) There is a one-to-one correspondence between left group-like elements $g \in H$ and right $H$-coactions $\beta: H_{t} \rightarrow H_{t} \otimes H$ that satisfy

$$
\left(\operatorname{id}_{H_{t}} \otimes \varepsilon_{t}\right) \circ \beta=\left(\varepsilon_{t} \otimes \operatorname{id}_{H_{t}}\right) \circ \Delta \circ \varepsilon_{t} .
$$

The correspondence is given by $g=\varepsilon\left(1_{0}\right) 1_{1}$ and $\beta(z)=\varepsilon_{t}\left((z g)^{\prime}\right) \otimes(z g)^{\prime \prime}$.

Theorem 5.6. Let $f: H \rightarrow H^{\prime}$ be a homomorphism of WBAs. Then the induced functor $f^{*}: \mathcal{M}^{H} \rightarrow \mathcal{M}^{H^{\prime}}$ pushes forward some right $H$-comodule $N \in\left|\mathcal{M}^{H}\right|$ to the monoidal unit $\mathbb{1} \in\left|\mathcal{M}^{H^{\prime}}\right|$ if and only if $N \cong H_{s}^{g}$ for some group-like $g \in H$ that satisfies $f(g)=1$. This group-like element is given by $g=\left(\varepsilon \otimes \mathrm{id}_{H}\right) \circ \beta_{N} \circ \eta(1)=$ $\varepsilon\left(1_{0}\right) 1_{1} \in H$.

Proof. If $N \cong H_{S}^{g}$, then the coaction $\beta_{N}: H_{S} \rightarrow H_{S} \otimes H$ is given by $\beta_{N}(x)=$ $x^{\prime} \otimes\left(g x^{\prime \prime}\right)$ for all $x \in H_{s}$. The push-forward reads $\beta_{f^{*}(N)}=\left(\left.f\right|_{H_{S}} \otimes f\right) \circ \beta_{N} \circ$ $\left.f\right|_{H_{S}}{ }^{-1}: H_{s}^{\prime} \rightarrow H_{s}^{\prime} \otimes H^{\prime}$. A direct computation shows that, since $f(g)=1$, we have $\beta_{f^{*}(N)}(y)=y^{\prime} \otimes y^{\prime \prime}$ for all $y \in H_{s}^{\prime}$, i.e. $f^{*}(N) \cong \mathbb{1} \in\left|\mathcal{M}^{H^{\prime}}\right|$.

Conversely, let $f^{*}(N) \cong \mathbb{1} \in\left|\mathcal{M}^{H^{\prime}}\right|$, i.e. there is a coaction $\beta_{f^{*}(N)}: H_{s}^{\prime} \rightarrow$ $H_{s}^{\prime} \otimes H$ such that $y_{0} \otimes f\left(y_{1}\right)=y^{\prime} \otimes y^{\prime \prime}$ for all $y \in H_{s}^{\prime}$.

First, the original coaction coincides with $\beta_{N}=\left(\left.f\right|_{H_{s}}{ }^{-1} \otimes \mathrm{id}_{H}\right) \circ \beta_{f^{*}(N)} \circ$ $\left.f\right|_{H_{S}}: H_{S} \rightarrow H_{S} \otimes H$ which can be shown to satisfy $x_{0} \otimes \varepsilon_{S}\left(x_{1}\right)=x^{\prime} \otimes \varepsilon_{S}\left(x^{\prime \prime}\right)$ for all $x \in H_{s}$. By Proposition 5.5(1), $g=\left(\varepsilon \otimes \mathrm{id}_{H}\right) \circ \beta_{N} \circ \eta(1)$ is right group-like and $\beta_{N}(x)=x^{\prime} \otimes\left(g x^{\prime \prime}\right)$ for all $x \in H_{s}$.

Second, there is a coaction $\delta=\left.\left(\left(\left.\varepsilon_{t} \circ f\right|_{H_{S}}{ }^{-1}\right) \otimes \mathrm{id}_{H}\right) \circ f\right|_{H_{S}} \circ \bar{\varepsilon}_{s}: H_{t} \rightarrow H_{t} \otimes H$ which can be shown to satisfy $z_{0} \otimes \varepsilon_{t}\left(z_{1}\right)=\varepsilon_{t}\left(z^{\prime}\right) \otimes z^{\prime \prime}$ for all $z \in H_{t}$. By Proposition 5.5(2), $\left(\left(\left.\varepsilon \circ \varepsilon_{t} \circ f\right|_{H_{S}}{ }^{-1}\right) \otimes \mathrm{id}_{H}\right) \circ f_{H_{S}} \circ \bar{\varepsilon}_{s} \circ \eta(1)=g$ is left group-like as well.

5.2. Completing the characterization. In this subsection, we compute the kernel of the surjection $\bar{\pi}: H[\mathcal{E}, \mathcal{E}] \rightarrow H$ of Proposition 4.7 and arrive at our characterization of the universal coend $H=\operatorname{coend}(\mathcal{C}, \omega)$ in Theorem 5.7. The main technical result is the application of Theorem 5.6 to the homomorphism of WBAs $\bar{\pi}: H[\mathcal{E}, \mathscr{E}] \rightarrow H$. 
Theorem 5.7. Let $\mathcal{C}$ be an essentially small, finitely split semisimple, $k$-linear, additive, autonomous monoidal category such that $k$ is a field and $\operatorname{Hom}(X, Y)$ is finitedimensional over $k$ for all $X, Y \in|\mathcal{E}|$. We choose an object $M \in|\mathcal{E}|$ that generates $\mathcal{C}$. Let $\mathcal{G}$ be the dimension graph and $\mathcal{E}=\left(E^{(n)}\right)_{n \in \mathbb{N}_{0}}$ be an endomorphism system for $\mathcal{C}$ with respect to $M$. We use the map $\bar{\pi}: H[\mathcal{E}, \mathcal{E}] \rightarrow H$ of Proposition 4.7. Let $G$ be the set

$$
G=\{g \in H[\mathcal{E}, \mathcal{E}] \mid g \text { is group-like and } \bar{\pi}(g)=1\}
$$

and $I_{G}$ be the two-sided ideal generated by the set $\{g-1 \mid g \in G\}$. Then $\bar{\pi}$ induces an isomorphism of WBAs

$$
H[\mathcal{E}, \mathcal{E}] / I_{G} \cong H .
$$

Proof. If $g$ is group-like such that $\bar{\pi}(g)=1$, the two-sided ideal generated by $g-1$ is also a two-sided coideal. The quotient $\widetilde{H}=H[\mathcal{E}, \mathcal{E}] / I_{G}$ is therefore a WBA. The map $\bar{\pi}: H[\mathcal{E}, \mathcal{E}] \rightarrow H$ obviously factors through this quotient and yields another surjection of WBAs $\tilde{\pi}: \widetilde{H} \rightarrow H$. We have to show that this map $\tilde{\pi}$ is injective.

We know from Proposition 4.12(5) that $H[\mathcal{E}, \mathcal{E}]$ is a coproduct of matrix coalgebras. We first show that $\widetilde{H}$ is a coproduct of matrix coalgebras as well, and then examine the action of $\tilde{\pi}$ on these matrix coalgebras in order to establish the injectivity of $\tilde{\pi}$. In the following, we denote by $p$ the canonical projection in the commutative diagram

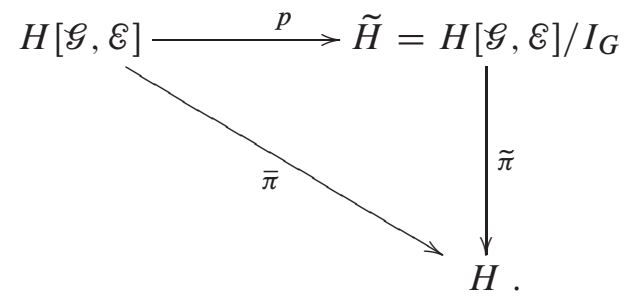

Let $V \in\left|\mathcal{M}^{H[\mathscr{E}, \mathcal{E}]}\right|$ be simple and $C(V)=V^{*} \otimes V \subseteq H[\mathcal{E}, \mathcal{E}]$ be the associated matrix coalgebra. We know from the proof of Proposition 4.12(5) that the restriction $\left.\bar{\pi}\right|_{C(V)}$ is injective, i.e. $\left.p\right|_{C(V)}$ is injective as well. Since $p$ is surjective, $\widetilde{H}$ is spanned by matrix coalgebras of the form $p(C(V)), V \in\left|\mathcal{M}^{H[\mathscr{\varepsilon}, \mathcal{E}]}\right|$.

Let now $W \in\left|\mathcal{M}^{H}\right|$ be simple and $C(W) \cong W^{*} \otimes W \subseteq H$. By Proposition 4.12(5), its pre-image $\bar{\pi}^{-1}(C(W)) \subseteq H[\mathcal{G}, \mathcal{E}]$ is a finite direct sum of matrix coalgebras. For each of these matrix coalgebras $C(V)=V^{*} \otimes V \subseteq H[\mathcal{E}, \mathcal{E}]$, the restriction $\left.p\right|_{C(V)}$ is injective, and so $p\left(\bar{\pi}^{-1}(C(W))\right.$ is a direct sum of (perhaps a smaller number of) matrix coalgebras. Since $p$ is surjective, $\tilde{\pi}^{-1}(C(W))=p\left(\bar{\pi}^{-1}(C(W))\right)$, i.e. the pre-image of $C(W)$ under $\tilde{\pi}$ is a finite direct sum of matrix coalgebras. In order to establish that $\tilde{\pi}$ is injective, it therefore suffices to show that this finite direct sum consists of one term only.

Recall that every potential term in the direct sum $\tilde{\pi}^{-1}(C(W))$ is of the form $p\left(V^{*} \otimes V\right)$ for some simple $V \in\left|\mathcal{M}^{H[\mathscr{\varepsilon}, \mathcal{E}]}\right|$, and so $V$ appears as a subcomodule of some $(\omega M)^{\widehat{\otimes} m}, m \geq 0$. We therefore need to prove the following: 
Let $X \subseteq(\omega M)^{\hat{\otimes} m}$ and $Y \subseteq(\omega M)^{\hat{\otimes} \ell}, m, \ell \in \mathbb{N}_{0}$, be simple right $H[\mathscr{E}, \mathcal{E}]$-comodules. If $X \cong Y$ as $H$-comodules (under push-forward by $\bar{\pi}$ ), then $X \cong Y$ as $\widetilde{H}$-comodules (under push-forward by $p$ ).

Let $X \cong Y$ be isomorphic as $H$-comodules. Recall that if $m=\ell$, Proposition 4.12(4) implies that $X \cong Y$ as $H[\mathscr{E}, \mathcal{E}]$-comodules and therefore as $\widetilde{H}$-comodules as well. We still have to deal with the case $m \neq \ell$.

Since $H$ is a WHA, $X^{*} \subseteq(\omega M)^{\hat{\otimes} t}$ for some $t \in \mathbb{N}_{0}$. There is an $H$-comodule

$$
T \subseteq X \hat{\otimes} X^{*} \subseteq(\omega M)^{\hat{\otimes}(m+t)},
$$

such that $T \cong H_{S}$ as $H$-comodules. Since $X \cong Y$ as $H$-comodules, there is another $H$-comodule

$$
\widetilde{T} \subseteq Y \hat{\otimes} X^{*} \subseteq(\omega M)^{\hat{\otimes}(\ell+t)},
$$

such that $\widetilde{T} \cong H_{s}$ as $H$-comodules. Both $T$ and $\widetilde{T}$ are also $H[\mathcal{E}, \mathcal{E}]$-comodules that are pushed-forward under $\bar{\pi}$ to the monoidal unit of $\mathcal{M}^{H}$. By Theorem 5.6, $T \cong H[\mathscr{E}, \mathcal{E}]_{s}^{g}$ and $\widetilde{T} \cong H[\mathscr{E}, \mathcal{E}]_{s}^{\tilde{g}}$ for some group-like $g, \widetilde{g} \in G$. Therefore, $T \cong \widetilde{H}_{s} \cong \widetilde{T}$ as $\widetilde{H}$-comodules.

On the other hand,

$$
\begin{aligned}
& X \hat{\otimes} \widetilde{T} \subseteq X \hat{\otimes}\left(Y \hat{\otimes} X^{*}\right) \subseteq(\omega M)^{\hat{\otimes}(m+\ell+t)}, \\
& Y \hat{\otimes} T \subseteq Y \hat{\otimes}\left(X \hat{\otimes} X^{*}\right) \subseteq(\omega M)^{\hat{\otimes}(\ell+m+t)}
\end{aligned}
$$

are isomorphic as $H$-comodules, and so by Proposition 4.12(2), also as $H[\mathcal{E}, \mathcal{E}]$-comodules and therefore as $\widetilde{H}$-comodules. We conclude that as $\widetilde{H}$-comodules,

$$
X \cong X \hat{\otimes} \widetilde{H}_{s} \cong X \hat{\otimes} \widetilde{T} \cong Y \hat{\otimes} T \cong Y \hat{\otimes} \widetilde{H}_{s} \cong Y .
$$

Note that since $H$ is a WHA, the left hand side of (5.7) becomes a WHA as well. Finally, we provide some additional details of the construction.

\subsection{Some further details}

Lemma 5.8. Under the assumptions of Theorem 5.7, let $g, \tilde{g} \in H[\mathcal{E}, \mathcal{E}]$ be group-like such that $\bar{\pi}(g)=1=\bar{\pi}(\tilde{g})$. Let $H[\mathscr{G}, \mathcal{E}]_{S}^{g} \subseteq(\omega M)^{\widehat{\otimes} m}$ and $H[\mathscr{G}, \mathcal{E}]_{S}^{\tilde{g}} \subseteq(\omega M)^{\hat{\otimes} n}$ for some $m, n \in \mathbb{N}_{0}$. If $m=n$, then $g=\tilde{g}$.

Proof. Recall from Proposition 4.12(5) that $H[\mathscr{E}, \mathcal{E}]$ is a coproduct of matrix coalgebras $C(V)=V^{*} \otimes V$ each of which is associated with a subcomodule $V \subseteq(\omega M)^{\hat{\otimes} m}$ for some $m \geq 0$, i.e. all comodules $H[\mathcal{E}, \mathcal{E}]_{s}^{g}$ are subcomodules of the form assumed.

Let now $m=n$. Pushing-forward these comodules along $\bar{\pi}$ yields isomorphic $H$-comodules $H_{s}^{\pi(g)} \cong H_{s} \cong H_{s}^{\pi(\tilde{g})}$. By Proposition 4.12(4), $H[\mathcal{E}, \mathcal{E}]_{s}^{g} \cong$ $H[\mathcal{E}, \mathcal{E}]_{S}^{\tilde{g}}$ are also isomorphic as $H[\mathcal{E}, \mathcal{E}]$-comodules. 
We now apply Theroem 5.6 to the homomorphism of WBAs $\bar{\pi}: H[\mathcal{E}, \mathcal{E}] \rightarrow H$ and choose $N=H[\mathcal{E}, \mathcal{E}]^{\tilde{g}}$. We know that $N$ pushes forward to the monoidal unit of $\mathcal{M}^{H}$ under $\bar{\pi}$ and that it is isomorphic as an $H[\mathcal{E}, \mathcal{E}]$-comodule to $H[\mathcal{E}, \mathcal{E}]_{S}^{g}$ where $g$ is group-like with $\bar{\pi}(g)=1$. Theorem 5.6 then gives a formula for $g$ in terms of the coaction of $H[\mathcal{G}, \mathcal{E}]_{S}^{\tilde{g}}$ :

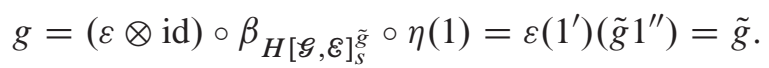

Proposition 5.9. Under the assumptions of Theorem 5.7, if in addition $C$ satisfies the strong Schur-Weyl property, then each $g \in G$ is central in $H[\mathcal{E}, \mathcal{E}]$ and satisfies $g=X(g)$ where $X(g)=\varepsilon_{t}\left(\varepsilon_{s}\left(g^{\prime}\right)\right) g^{\prime \prime} \varepsilon_{s}\left(\varepsilon_{t}\left(g^{\prime \prime}\right)\right)$. Here, $X$ acts on homogeneous elements $[p \mid q]_{m} \in H[\mathcal{E}, \mathcal{E}]_{m}, m \in \mathbb{N}_{0}$, as follows:

$$
X\left([p \mid q]_{m}\right)=\delta_{\sigma(p), \tau(p)} \delta_{\sigma(q), \tau(q)}[p \mid q]_{m} .
$$

Furthermore, using the surjection $\pi_{R}: H[\mathcal{E}, R] \rightarrow H$ of WBAs of Proposition 4.10, each $g \in G_{R}$ with

$$
G_{R}=\left\{g \in H[\mathcal{E}, R] \mid g \text { is group-like and } \pi_{R}(g)=1\right\}
$$

is central in $H[\mathcal{E}, R]$.

Proof. We abbreviate $N=H[\mathcal{E}, \mathcal{E}]_{s}$ and $N^{g}=H[\mathcal{E}, \mathcal{E}]_{S}^{g}$ and consider the coactions

$$
\begin{aligned}
\beta_{M}: M & \longrightarrow M \otimes H[\mathcal{E}, \mathcal{E}], \\
p & \longmapsto \sum_{q \in \mathscr{E}^{1}} q \otimes[q \mid p]_{1},
\end{aligned}
$$

and

$$
\begin{aligned}
\beta_{N^{g}}: N^{g} & \longrightarrow N^{g} \otimes H[\mathcal{E}, \mathcal{E}], \\
j & \longmapsto \sum_{\ell \in \mathscr{E}^{0}} \ell \otimes\left(g[\ell \mid j]_{0}\right),
\end{aligned}
$$

for $p \in \mathscr{E}^{1}, j \in \mathscr{\ell}^{0}$. Applying $\mathrm{id}_{N^{g}} \otimes \mathrm{id}_{M} \otimes \bar{\pi}$ to both sides of the following equation

$$
\beta_{N^{g} \hat{\otimes} M}(j \otimes p)=\left(\sigma_{N^{g}, M}^{-1} \otimes \operatorname{id}_{H[\mathscr{E}, \mathcal{E}]}\right) \circ \beta_{M \hat{\otimes}_{N^{g}}} \circ \sigma_{N^{g}, M}(j \otimes p),
$$

exploiting that $\bar{\pi}(g)=1$ and that $\bar{\pi}$ is injective on $H[\mathcal{E}, \mathcal{E}]_{0} \cdot H[\mathcal{E}, \mathcal{E}]_{1} \subseteq H[\mathcal{E}, \mathcal{E}]_{1}$ yields

$$
\beta_{N \hat{\otimes} M}=\left(\sigma_{N^{g}, M}^{-1} \otimes \operatorname{id}_{H[\mathcal{E}, \mathcal{E}]}\right) \circ \beta_{M \widehat{\otimes} N} \circ \sigma_{N^{g}, M}
$$

where $\beta_{N \widehat{\otimes} M}$ and $\beta_{M \widehat{\otimes} N}$ contain the coaction of the monoidal unit,

$$
\begin{aligned}
\beta_{N}: N^{g} & \longrightarrow N^{g} \otimes H[\mathcal{E}, \mathcal{E}] \\
j & \longmapsto \sum_{\ell \in \mathcal{E}^{0}} \ell \otimes[\ell \mid j]_{0} .
\end{aligned}
$$


Applying $(-\otimes-\otimes(g \cdot-))$ to (16), pre- and post-composing with $\sigma_{N^{g}, M}^{-1}$ and $\sigma_{N^{g}, M} \otimes \operatorname{id}_{H[\mathscr{E}, \mathcal{E}]}$, and then pre- and post-composing with $\rho_{M}$ and $\rho_{M}^{-1}$, respectively, allows us to compute

$$
\sum_{q \in \mathscr{S}^{1}} q \otimes\left(g[q \mid p]_{1}\right)=\sum_{q \in \mathscr{G}^{1}} q \otimes\left([q \mid p]_{1} X(g)\right) .
$$

Now we repeat all of the above argument for $\mathbb{1}=H[\mathcal{E}, \mathcal{E}]_{S}$ rather than $M$. In this case, we exploit the fact that $\bar{\pi}$ is injective on $H[\mathscr{E}, \mathcal{E}]_{0} \cdot H[\mathcal{E}, \mathcal{E}]_{0} \subseteq H[\mathcal{E}, \mathscr{E}]_{0}$ and obtain that for all $\ell \in \mathscr{E}^{0}$ :

$$
\sum_{j \in \mathscr{E}^{0}} j \otimes\left(g[j \mid \ell]_{0}\right)=\sum_{j \in \mathscr{S}^{0}} j \otimes\left([j \mid \ell]_{0} X(g)\right) .
$$

Since $\ell$ satisfies the strong Schur-Weyl property, $\mathbb{1}$ is simple and therefore the $[j \mid \ell]_{0}$, $j, \ell \in \mathscr{E}^{0}$, form a basis of $(\omega M)^{\hat{\otimes} 0}$, and so comparing coefficients yields

$$
g[j \mid \ell]_{0}=[j \mid \ell]_{0} X(g)
$$

for all $j, \ell \in \mathscr{E}^{0}$. Since $\eta(1) \in H[\mathcal{E}, \mathcal{E}]_{0}$, we conclude that $g=X(g)$.

Finally, since $M$ is simple, the $[p \mid q]_{1}, p, q \in \mathcal{E}^{1}$, form a basis of $\omega M$, and so we can compare coefficients in (17) and find that

$$
g[p \mid q]_{1}=[p \mid q]_{1} g
$$

for all $p, q \in \mathscr{E}^{1}$. Since $H[\mathcal{E}, \mathcal{E}]$ as an algebra is generated by $H[\mathcal{E}, \mathcal{E}]_{0} \cup H[\mathcal{E}, \mathcal{E}]_{1}$, $g$ is central. The argument for $G_{R}$ and $\pi_{R}$ is identical.

In order to compute $X(g)$ on homogeneous elements, we note that

$$
\begin{aligned}
\varepsilon_{s}\left([p \mid q]_{m}\right) & =\delta_{p q} \sum_{j \in \mathscr{E}^{0}}[j \mid \sigma(p)]_{0}, \\
\varepsilon_{t}\left([p \mid q]_{m}\right) & =\delta_{p q} \sum_{j \in \mathscr{E}^{0}}[\tau(q) \mid j]_{0}, \\
\varepsilon_{t}\left(\varepsilon_{s}\left([p \mid q]_{m}\right)\right. & =\delta_{p q} \sum_{j \in \mathscr{E}^{0}}[\sigma(p) \mid j]_{0}, \\
\varepsilon_{s}\left(\varepsilon_{t}\left([p \mid q]_{m}\right)\right. & =\delta_{p q} \sum_{j \in \mathscr{E}^{0}}[j \mid \tau(q)]_{0},
\end{aligned}
$$

from which the claim follows in a direct computation.

The following proposition is useful if one wishes to determine the relevant grouplike elements for a given category $\ell$. 
Proposition 5.10. Under the assumptions of Theorem 5.7 with the set $G$ of (14), the following holds.

(1) For each $m \in \mathbb{N}_{0}$, the set $G \cap H[\mathcal{E}, \mathcal{E}]_{m}$ contains at most one element which we denote by $g_{m}$ if it exists.

(2) For the elements of Part (1), we have $g_{m} g_{\ell}=g_{m+\ell}$ for all $m, \ell$.

(3) $G$ is an abelian monoid.

(4) Each $g \in G, g \neq 1$, has infinite order.

Proof. (1) Let $g, g^{\prime} \in G \cap H[\mathcal{E}, \mathcal{E}]_{m}$ for some $m \in \mathbb{N}_{0}$. Then by Theorem 5.6 and by the grading of $H[\mathscr{E}, \mathcal{E}]$, both $H[\mathscr{E}, \mathscr{E}]_{S}^{g} \subseteq(\omega M)^{\hat{\otimes} m}$ and $H[\mathscr{E}, \mathscr{E}]_{S}^{g^{\prime}} \subseteq(\omega M)^{\hat{\otimes} m}$. Lemma 5.8 implies $g=g^{\prime}$.

(2) By the grading, $g_{m} g_{\ell} \in H[\mathscr{G}, \mathcal{E}]_{m+\ell}$ and $g_{m} g_{\ell}$ is group-like with $\bar{\pi}\left(g_{m} g_{\ell}\right)=$ 1. Part (1) then implies the claim.

(3) Because $\bar{\pi}$ is a homomorphism of unital associative algebras and because of Part (2).

(4) Because of Parts (1) and (2) and the fact that the unit is in degree zero, $1 \in H[\mathcal{E}, \mathcal{E}]_{0}$.

Proposition 5.11. Under the assumptions of Theorem 5.7, if $\mathcal{C}$ is braided, the WBA $H[\mathcal{E}, \mathcal{E}] / I_{G}$ is coquasi-triangular with the universal $r$-form induced from $H[\mathcal{E}, \mathcal{E}]$, and the isomorphism (5.7) is an isomorphism of coquasi-triangular WBAs.

Proof. The coquasi-triangular structure of $H[\mathcal{E}, \mathcal{E}]$ descends to $H[\mathcal{E}, \mathcal{E}] / I_{G}$ provided that the universal $r$-form $r: H[\mathscr{G}, \mathcal{E}] \otimes H[\mathscr{G}, \mathcal{E}] \rightarrow k$ and its weak convolution inverse $\bar{r}: H[\mathcal{E}, \mathcal{E}] \otimes H[\mathscr{E}, \mathcal{E}] \rightarrow k$ satisfy

$$
r\left(I_{G}, H[\mathcal{E}, \mathcal{E}]\right)=0=r\left(H[\mathcal{E}, \mathcal{E}], I_{G}\right), \quad \bar{r}\left(I_{G}, H[\mathcal{E}, \mathcal{E}]\right)=0=\bar{r}\left(H[\mathcal{E}, \mathcal{E}], I_{G}\right) .
$$

This holds on the generators $1-g, g \in G$, of $I_{G}$ because $\bar{\pi}$ is a homomorphism of coquasi-triangular WBAs, $\bar{\pi}(g)=1$ and $r(1,-)=\varepsilon(-)=r(-, 1)$ and $\bar{r}(1,-)=\varepsilon(-)=\bar{r}(-, 1)$. It extends to the two-sided ideal by (1) and (2). The isomorphism (5.7) is one of coquasi-triangular WHAs because $\bar{\pi}$ pushes forward all relevant comodules.

\section{Examples}

6.1. The modular categories associated with $U_{q}\left(\mathfrak{s l}_{2}\right)$. In this section, we review the modular categories associated with $U_{q}\left(\mathfrak{s l}_{2}\right)$ at suitable roots of unity following [8] and present them as the categories of finite-dimensional comodules of a WHA $H[\mathcal{E}, \mathcal{E}] / I_{G}$. 
6.1.1. Description of the categories. Let $r \in\{2,3,4, \ldots\}$ and $A$ be a primitive $4 r$-th root of unity, $q=A^{2}$. For simplicity, we work over the complex numbers $k=\mathbb{C}$. The morphisms of our category $\mathcal{C}$ are represented by plane projections of oriented framed tangles, drawn in blackboard framing. The coherence theorem for ribbon categories [19] ensures that each diagram defines a morphism of $\mathcal{C}$. Since $\mathcal{C}$ is $k$-linear, we can take formal linear combinations of diagrams with coefficients in $k$. All our diagrams are read from top to bottom.

The braiding of $\mathcal{C}$ is such that a crossing in our plane projections can be resolved using the recursion relation for the Kauffman bracket

$$
\lambda^{\prime}=\overbrace{}^{-}+A^{-1})\left(, \bigcirc=-\left(q+q^{-1}\right),\right.
$$

ignoring the orientations for now. The Jones-Wenzl idempotents $P_{n}, 1 \leq n \leq r-2$, are formal linear combinations of planar $(n, n)$-tangles that can be defined recursively by

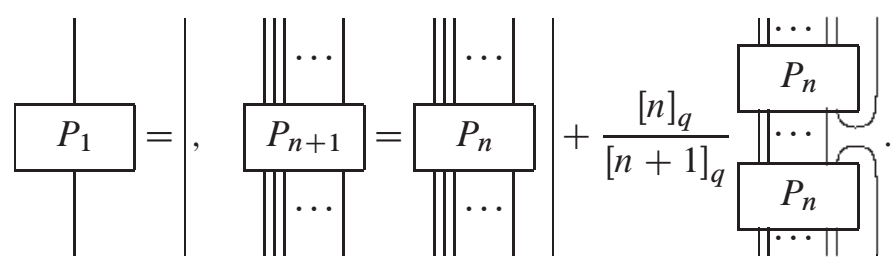

where $[n]_{q}=\left(q^{n}-q^{-n}\right) /\left(q-q^{-1}\right), n \in \mathbb{Z}$, are the quantum integers. The isomorphism classes of simple objects of $\mathcal{C}$ are indexed by the set $I=\{0,1, \ldots, r-2\}$. The identity morphism of the object $V_{n}, n \in I$, is the identity $(n, n)$-tangle with the idempotent $P_{n}$ inserted somewhere (anywhere). As a shortcut, we write a single line labeled by $n$,

$$
\left.\right|^{n}=\underset{|||\cdots|}{P_{n}} .
$$

The object $V_{0}$ indexed by $0 \in I$ is the monoidal unit and can be made invisible in our diagrams thanks to the coherence theorem. The categorical dimension of the simple objects is given by

$$
\Delta_{n}=\bigcirc^{n}=(-1)^{n}[n+1]_{q},
$$

which is non-zero for all $n \in I$.

Two special features of $U_{q}\left(\mathfrak{s l}_{2}\right)$ are exploited. First, the simple objects are isomorphic to their duals, and the choice of representatives $V_{j}, j \in I$, of the simple objects is such that $\left(V_{j}\right)^{*}=V_{j}$ are equal rather than merely isomorphic. This allows us to omit any arrows from the diagrams that would indicate the orientation of the ribbon tangle.

Second, there are no higher multiplicities, i.e. for all $a, b, c \in I$, we have $\operatorname{dim}_{k} \operatorname{Hom}\left(V_{a} \otimes V_{b}, V_{c}\right) \in\{0,1\}$. More precisely, $\operatorname{Hom}\left(V_{a} \otimes V_{b}, V_{c}\right) \cong k$ if and only if the triple $(a, b, c)$ is admissible. Otherwise, $\operatorname{Hom}\left(V_{a} \otimes V_{b}, V_{c}\right)=\{0\}$. 
Definition 6.1. A triple $(a, b, c) \in I^{3}$ is called admissible if the following conditions hold.

(1) $a+b+c \equiv 0 \bmod 2$ (parity)

(2) $a+b-c \geq 0$ and $b+c-a \geq 0$ and $c+a-b \geq 0$ (quantum triangle inequality),

(3) $a+b+c \leq 2 r-4$ (non-negligibility).

A special choice of basis vector of $\operatorname{Hom}\left(V_{a}, V_{b} \otimes V_{c}\right)$ is denoted by a trivalent vertex:

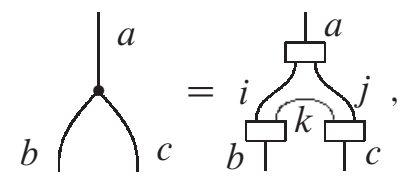

where $i=(a+b-c) / 2, j=(a+c-b) / 2$ and $k=(b+c-a) / 2$ and the boxes denote Jones-Wenzl idempotents. If we draw such a diagram for a triple $(a, b, c) \in I^{3}$ that is not admissible, then by convention, we multiply the entire diagram by zero. We also need the theta graph

$$
\vartheta(a, b, c)=\bigodot_{c}^{a},
$$

which is non-zero for all admissible triples $(a, b, c)$. When we compose the morphisms associated with such diagrams, the composition is zero unless the labels at the open ends of the tangles match, i.e. putting

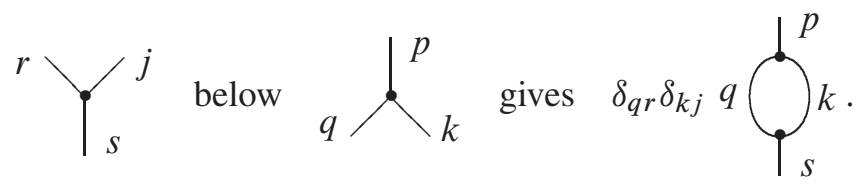

Exploiting semi-simplicity and Schur's lemma, we compute

$$
q \bigcap_{s}^{p} k=\left.\delta_{p s} \frac{\vartheta(p, q, k)}{\Delta_{p}}\right|^{p} .
$$

The quantum $6 j$-symbol is defined as

$$
\left\{\begin{array}{lll}
a & b & i \\
c & d & j
\end{array}\right\}_{q}=\frac{\Delta_{i}}{\vartheta(a, d, i) \vartheta(b, c, i)} i
$$


It is used in the recoupling identity,

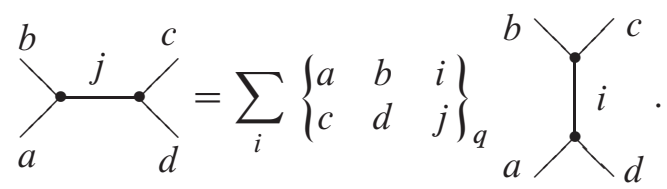

6.1.2. The dimension graph. We now assume that $r \geq 3$. The category $e$ is generated by $M=V_{1}$ (Definition 3.4). From the decompositions $V_{0} \otimes M \cong V_{1}$, $V_{j} \otimes M \cong V_{j-1} \oplus V_{j+1}$ for all $1 \leq j \leq r-3$ and $V_{r-2} \otimes M \cong V_{r-3}$, we obtain the dimension graph $\mathcal{E}$ of $\mathcal{C}$ with respect to $M$ (Definition 3.6):

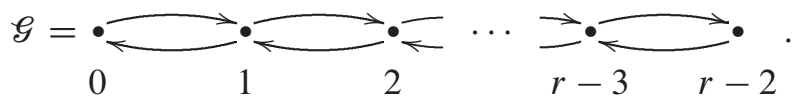

Since for any two vertices $j, \ell \in \ell^{0}=I$, there is at most one edge from $j$ to $\ell$, we specify a path $p \in \mathcal{E}^{m}$ of length $m \in \mathbb{N}_{0}$ by the sequence of the $m+1$ vertices along $p$, i.e. $p=\left(i_{0}, \ldots, i_{m}\right) \in I^{m+1}$. The source and target of this path are $\sigma(p)=i_{m}$ and $\tau(p)=i_{0}$.

At this point, the reader should be familiar with the WBA $H[\mathcal{E}]$ associated with the graph $\mathcal{E}$ (Proposition 3.1). As an algebra, $H[\mathcal{E}] \cong k(\mathcal{E} \times \mathscr{E}$ ) is the path algebra of the quiver $\mathscr{E} \times \mathcal{E}$. As a coalgebra, it is a direct sum of matrix coalgebras: one for each degree, i.e. for each length of paths. Our construction shows that the category $\zeta$ is equivalent to the category of finite-dimensional comodules of $H$ [ $\mathcal{E}]$ modulo the relations (19) and (21) below.

6.1.3. The fundamental surjection. We use the same basis of $\omega M=\operatorname{Hom}(\widehat{V}$, $\widehat{V} \otimes M)$ as in [16] and [17], i.e. $\left\{e_{i, i+1}^{(M)}, e_{i+1, i}^{(M)} \mid 0 \leq i \leq r-3\right\}$ which reads in terms of diagrams,

$$
e_{i, i+1}^{(M)}=\sum_{i+1}^{i} \text { and } e_{i+1, i}^{(M)}=\sum_{i}^{i+1} .
$$

The surjection $\pi: H[\mathcal{E}] \rightarrow H=\operatorname{coend}(\mathcal{C}, \omega)$ (Theorem 3.8) then maps

$$
\begin{aligned}
\pi\left([(j) \mid(\ell)]_{0}\right) & =\left[e_{(\mathbb{1})}^{j} \mid e_{\ell}^{(\mathbb{1})}\right]_{\mathbb{1}}, \\
\pi\left(\left[\left(j_{0}, j_{1}\right) \mid\left(\ell_{0}, \ell_{1}\right)\right]_{1}\right) & =\left[e_{(M)}^{j_{0}, j_{1}} \mid e_{\ell_{1}, \ell_{0}}^{(M)}\right]_{M},
\end{aligned}
$$

for $j, j_{0}, j_{1}, \ell, \ell_{0}, \ell_{1} \in I$ and $j_{1}=j_{0} \pm 1, \ell_{1}=\ell_{0} \pm 1$. We refer to the explanations preceding Theorem 3.8 for the bases used on the right hand side.

6.1.4. The endomorphism adapted WBA. The category $C$ satisfies the strong Schur-Weyl property (Definition 4.2), i.e. the monoidal unit $\mathbb{1}=V_{0}$ and the generating object $M=V_{1}$ are both simple, and the endomorphism algebras $\operatorname{End}\left(M^{\otimes m}\right)$, $m \geq 2$, are generated by braiding and inverse braiding of adjacent tensor factors. 
The ideal $I_{\mathscr{E}}$ in the definition of the endomorphism adapted WBA $H[\mathcal{E}, \mathcal{E}]=$ $H[\mathcal{E}] / I_{\mathscr{E}}$ (Definitions 4.5 and 4.8 ) is therefore generated by homogeneous relations of degree two:

$$
\begin{aligned}
& \sum_{\left(i_{0}, i_{1}, i_{2}\right) \in \mathcal{G}^{2}}\left[\left(j_{0}, j_{1}, j_{2}\right) \mid\left(i_{0}, i_{1}, i_{2}\right)\right]_{2} R_{\left(i_{0}, i_{1}, i_{2}\right) ;\left(\ell_{0}, \ell_{1}, \ell_{2}\right)} \\
& \quad-\sum_{\left(i_{0}, i_{1}, i_{2}\right) \in \mathcal{E}^{2}} R_{\left(j_{0}, j_{1}, j_{2}\right) ;\left(i_{0}, i_{1}, i_{2}\right)}\left[\left(i_{0}, i_{1}, i_{2}\right) \mid\left(\ell_{0}, \ell_{1}, \ell_{2}\right)\right]_{2},
\end{aligned}
$$

for all paths of length two $\left(j_{0}, j_{1}, j_{2}\right) \in \mathcal{E}^{2}$ and $\left(\ell_{0}, \ell_{1}, \ell_{2}\right) \in \mathcal{E}^{2}$. Note that these relations are non-trivial only if $j_{0}=\ell_{0}$ and $j_{2}=\ell_{2}$ (Remark 4.4(2)).

A direct computation using Temperley-Lieb recoupling calculus yields the following non-zero coefficients:

$$
\begin{aligned}
R_{(j, j \pm 1, j) ;(j, j \pm 1, j)} & =\mp q^{-1 / 2} \frac{q^{ \pm(j+1)}}{[j+1]_{q}}, \\
R_{(j, j-1, j) ;(j, j+1, j)} & =q^{-1 / 2} \frac{[j]_{q}[j+2]_{q}}{[j+1]_{q}^{2}} \\
R_{(j, j+1, j) ;(j, j-1, j)} & =q^{-1 / 2} \\
R_{(j, j \pm 1, j \pm 2) ;(j, j \pm 1, j \pm 2)} & =q^{-3 / 2}
\end{aligned}
$$

6.1.5. The relevant group-like elements. In order to compute the kernel $I_{G}$ of the induced surjection $\bar{\pi}: H[\mathcal{E}, \mathcal{E}] \rightarrow H$, we systematically consider the simple comodules of $H$ and $H[\mathcal{E}, \mathcal{E}]$, proceeding by increasing degree according to the tensor power $m$ of $M^{\otimes m}, m \in \mathbb{N}_{0}$.

In the following table, we show the decomposition of $(\omega M)^{\hat{\otimes} m}$ as an $H$-comodule which is known from $\mathcal{C}$, and the decomposition of $k^{m}$ as an $H[\mathcal{E}, \mathcal{E}]$-comodule which follows from Proposition 4.12(4). Assume for now that $r$ is big.

\begin{tabular}{r|l|l}
$m$ & $(\omega M)^{\widehat{\otimes} m} \in\left|\mathcal{M}^{H}\right|$ & $k \mathscr{E}^{m} \in\left|\mathcal{M}^{H[\mathcal{E}, \mathcal{E}]}\right|$ \\
\hline 0 & $V_{0}$ & $V_{0}$ \\
1 & $V_{1}$ & $V_{1}$ \\
2 & $V_{2} \oplus V_{0}$ & $V_{2} \oplus V_{0}^{\prime}$ \\
3 & $V_{3} \oplus 2 V_{1}$ & $V_{3} \oplus 2 V_{1}^{\prime}$ \\
4 & $V_{4} \oplus 3 V_{2} \oplus 2 V_{0}$ & $V_{4} \oplus 3 V_{2}^{\prime} \oplus 2 V_{0}^{\prime \prime}$ \\
$\ldots$ & $\ldots$ & $\ldots$
\end{tabular}

For each $j \in \mathrm{I}$, the objects $V_{j}, V_{j}^{\prime}, V_{j}^{\prime \prime}, \ldots$ are simple $H[\mathcal{G}, \mathcal{E}]$-comodules that are pairwise non-isomorphic as $H[\mathscr{E}, \mathcal{E}]$-comodules but that are all pushed forward to the $H$-comodule $V_{j}$ under $\bar{\pi}$. Note that we have suppressed the long forgetful functor and written $V_{j}$ for $\omega V_{j}$. 
We see that $m=2$ is the smallest degree in which there is an $H[\mathcal{E}, \mathscr{E}]$-comodule, $V_{0}^{\prime}$, which is not isomorphic to $V_{0}$, but pushed forward to it under $\bar{\pi}$. Therefore, by Theorem 5.6, $V_{0}^{\prime}$ is characterized by a group-like element $g_{2} \in H[\mathscr{E}, \mathcal{E}]_{2}$ for which $\bar{\pi}\left(g_{2}\right)=1$. Upon dividing $H[\mathcal{E}, \mathcal{E}]$ by the relation $g_{2}-1, V_{0}^{\prime}$ and $V_{0}$ will become isomorphic.

The next higher degree with an $H[\mathscr{E}, \mathcal{E}]$-comodule non-isomorphic, but pushed forward to $V_{0}$ is $m=4$. Since the group-like $g_{2}^{2}$ is of degree $m=4$ and satisfies $\bar{\pi}\left(g_{2}\right)=1$, by Proposition 5.10, $g_{2}^{2}$ is the group-like that characterizes $V_{0}^{\prime \prime}$. Notice that the quotient by $g_{2}-1$ will also render $V_{1}^{\prime}$ isomorphic to $V_{1}, V_{0}^{\prime \prime}$ isomorphic to $V_{0}$ and $V_{2}^{\prime}$ isomorphic to $V_{2}$.

If $r$ is not large enough, the above argument is unchanged except that some of the 'biggest' comodules are absent from the decompositions. The pattern continues in higher degrees, and the only relevant group-like is $g_{2}$.

In order to compute $g_{2}$, we explicitly decompose $\omega M \hat{\otimes} \omega M \cong V_{2} \oplus V_{0}^{\prime}$ and compute $g_{2}$ as the group-like that characterizes $V_{0}^{\prime}$ from Theorem 5.6. For the decomposition, we calculate the idempotent $Q=\omega\left(\operatorname{id}_{M \otimes M}-P_{2}\right)$ associated with $V_{0}^{\prime}$. Here, $P_{2}$ is the Jones-Wenzl idempotent of $V_{2} \subseteq M \otimes M . Q$ takes non-zero values in the following cases:

$$
\begin{aligned}
Q((0,1,0))= & (0,1,0), \\
Q((j, j+1, j))= & \frac{[j+2]_{q}}{[2]_{q}[j+1]_{q}}(j, j+1, j) \\
& -\frac{[j]_{q}[j+2]_{q}}{[2]_{q}[j+1]_{q}{ }^{2}}(j, j-1, j), \\
Q((j, j-1, j))= & -\frac{1}{[2]_{q}}(j, j+1, j) \\
& +\frac{[j]_{q}}{[2]_{q}[j+1]_{q}}(j, j-1, j), \\
Q((r-2, r-3, r-2))= & (r-2, r-3, r-2),
\end{aligned}
$$

for $1 \leq j \leq r-3$. A basis for $V_{0}^{\prime} \subseteq \omega M \widehat{\otimes} \omega M$ is therefore given by

$$
\begin{aligned}
b_{0} & =(0,1,0), \\
b_{j} & =\left([j+1]_{q}(j, j+1, j)-[j]_{q}(j, j-1, j)\right) / \sqrt{2}, \\
b_{r-2} & =-(r-2, r-3, r-2) .
\end{aligned}
$$

We use the coefficients of the comodule $V_{0}^{\prime}$ in this basis and compute the group-like 
to be

$$
\begin{aligned}
g_{2}=\sum_{j, \ell=0}^{r-2} \alpha_{j} \alpha_{\ell}\left(\frac{[\ell+1]_{q}}{[j}+1\right]_{q} & {[(j, j+1, j) \mid(\ell, \ell+1, \ell)]_{2} } \\
& +\frac{[\ell]_{q}}{[j]_{q}}[(j, j-1, j) \mid(\ell, \ell-1, \ell)]_{2} \\
& -\frac{[\ell+1]_{q}}{[j]_{q}}[(j, j-1, j) \mid(\ell, \ell+1, \ell)]_{2} \\
& \left.-\frac{[\ell]_{q}}{[j+1]_{q}}[(j, j+1, j) \mid(\ell, \ell-1, \ell)]_{2}\right),
\end{aligned}
$$

with $\alpha_{0}=\alpha_{r-2}=1$ and $\alpha_{j}=1 / \sqrt{2}$ for all $1 \leq j \leq r-3$. In (20) it is understood that terms with a path $(j, j \pm 1, j)$ are omitted from the expression whenever $j \pm 1<0$ or $j \pm 1>r-2$.

The kernel of $\bar{\pi}: H[\mathscr{E}, \mathscr{E}] \rightarrow H$ is therefore (Theorem 5.7) generated by

$$
1-g_{2} \text {. }
$$

We have shown that the category $C$ is equivalent to the category of finite-dimensional comodules of the quotient of $H[\mathscr{E}]$ for the graph $\mathscr{E}$ of (18) modulo the relations (19) and (21).

\section{A. Summary of notation and conventions}

In this appendix, we collect the relevant definitions and properties of monoidal, autonomous, braided monoidal and abelian categories, following Schauenburg [21] and Mac Lane [10].

\section{A.1. Monoidal categories}

Definition A.1. A monoidal category $(\mathcal{C}, \otimes, \mathbb{1}, \alpha, \lambda, \rho)$ is a category $\mathcal{C}$ with a bifunctor $\otimes: \mathcal{C} \times \mathcal{C} \rightarrow \mathcal{C}$ (tensor product), an object $\mathbb{1} \in|\mathcal{C}|$ (monoidal unit) and natural isomorphisms $\alpha_{X, Y, Z}:(X \otimes Y) \otimes Z \rightarrow X \otimes(Y \otimes Z)$ (associator), $\lambda_{X}: \mathbb{1} \otimes X \rightarrow X$ (left-unit constraint) and $\rho_{X}: X \otimes \mathbb{1} \rightarrow X$ (right-unit constraint) for all $X, Y, Z \in|\mathcal{C}|$, subject to the pentagon axiom

$$
\alpha_{X, Y, Z \otimes W} \circ \alpha_{X \otimes Y, Z, W}=\left(\operatorname{id}_{X} \otimes \alpha_{Y, Z, W}\right) \circ \alpha_{X, Y \otimes Z, W} \circ\left(\alpha_{X, Y, Z} \otimes \operatorname{id}_{W}\right)
$$

and the triangle axiom

$$
\rho_{X} \otimes \operatorname{id}_{Y}=\left(\operatorname{id}_{X} \otimes \lambda_{Y}\right) \circ \alpha_{X, \mathbb{1}, Y}
$$

for all $X, Y, Z, W \in|\mathcal{E}|$. 
Definition A.2. Let $(\mathcal{C}, \otimes, \mathbb{1}, \alpha, \lambda, \rho)$ and $\left(\boldsymbol{C}^{\prime}, \otimes^{\prime}, \mathbb{1}^{\prime}, \alpha^{\prime}, \lambda^{\prime}, \rho^{\prime}\right)$ be monoidal categories.

(1) A lax monoidal functor $\left(F, F_{X, Y}, F_{0}\right): \bigodot \rightarrow \ell^{\prime}$ consists of a functor $F: \ell \rightarrow$ $\ell^{\prime}$, morphisms $F_{X, Y}: F X \otimes \otimes^{\prime} F Y \rightarrow F(X \otimes Y)$ that are natural in $X, Y \in|\zeta|$, and

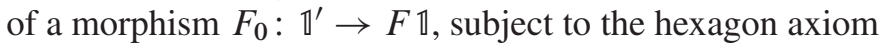

$$
F_{X, Y \otimes Z} \circ\left(\operatorname{id}_{F X} \otimes^{\prime} F_{Y, Z}\right) \circ \alpha_{F X, F Y, F Z}^{\prime}=F \alpha_{X, Y, Z} \circ F_{X \otimes Y, Z} \circ\left(F_{X, Y} \otimes^{\prime} \mathrm{id}_{F Z}\right)
$$

and the two squares

$$
\begin{aligned}
\lambda_{F X}^{\prime} & =F \lambda_{X} \circ F_{\mathbb{1}, X} \circ\left(F_{0} \otimes^{\prime} \operatorname{id}_{F X}\right), \\
\rho_{F X}^{\prime} & =F \rho_{X} \circ F_{X, \mathbb{1}} \circ\left(\operatorname{id}_{F X} \otimes^{\prime} F_{0}\right)
\end{aligned}
$$

for all $X, Y, Z \in|\varphi|$.

(2) An oplax monoidal functor $\left(F, F^{X, Y}, F^{0}\right): \bigodot \rightarrow \ell^{\prime}$ consists of a functor $F: \mathcal{C} \rightarrow \mathcal{C}^{\prime}$, morphisms $F^{X, Y}: F(X \otimes Y) \rightarrow F X \otimes^{\prime} F Y$ that are natural in $X, Y \in|\mathcal{E}|$, and of a morphism $F^{0}: F \mathbb{1} \rightarrow \mathbb{1}^{\prime}$, subject to the hexagon axiom

$$
\left(\operatorname{id}_{F X} \otimes^{\prime} F^{Y, Z}\right) \circ F^{X, Y \otimes Z} \circ F \alpha_{X, Y, Z}=\alpha_{F X, F Y, F Z}^{\prime} \circ\left(F^{X, Y} \otimes^{\prime} \operatorname{id}_{F Z}\right) \circ F^{X \otimes Y, Z}
$$

and the two squares

$$
\begin{aligned}
F \lambda_{X} & =\lambda_{F X}^{\prime} \circ\left(F^{0} \otimes^{\prime} \operatorname{id}_{F X}\right) \circ F^{\mathbb{1}, X}, \\
F \rho_{X} & =\rho_{F X}^{\prime} \circ\left(\operatorname{id}_{F X} \otimes^{\prime} F^{0}\right) \circ F^{X, \mathbb{1}}
\end{aligned}
$$

for all $X, Y, Z \in|\Gamma|$.

(3) A strong monoidal functor $\left(F, F_{X, Y}, F_{0}\right): e \rightarrow \ell^{\prime}$ is a lax monoidal functor such that all $F_{X, Y}, X, Y \in|\mathcal{E}|$ and $F_{0}$ are isomorphisms.

Definition A.3. Let $(\mathcal{C}, \otimes, \mathbb{1}, \alpha, \lambda, \rho)$ be a monoidal category. A left-dual $\left(X^{*}, \mathrm{ev}_{X}\right.$, $\operatorname{coev}_{X}$ ) of an object $X \in|\mathcal{C}|$ consists of an object $X^{*} \in|\mathcal{}|$ and morphisms $\mathrm{ev}_{X}: X^{*} \otimes X \rightarrow \mathbb{1}$ (left evaluation) and $\operatorname{coev}_{X}: \mathbb{1} \rightarrow X \otimes X^{*}$ (left coevaluation) that satisfy the triangle identities

$$
\begin{gathered}
\rho_{X} \circ\left(\mathrm{id}_{X} \otimes \mathrm{ev}_{X}\right) \circ \alpha_{X, X^{*}, X} \circ\left(\operatorname{coev}_{X} \otimes \mathrm{id}_{X}\right) \circ \lambda_{X}^{-1}=\mathrm{id}_{X}, \\
\lambda_{X^{*}} \circ\left(\mathrm{ev}_{X} \otimes \mathrm{id}_{X^{*}}\right) \circ \alpha_{X^{*}, X, X^{*}}^{-1} \circ\left(\operatorname{id}_{X^{*}} \otimes \operatorname{covv}_{X}\right) \circ \rho_{X^{*}}^{-1}=\mathrm{id}_{X^{*}} .
\end{gathered}
$$

If $\varphi$ is a monoidal category and $f: X \rightarrow Y$ a morphism of $\varphi$ such that both $X$ and $Y$ have left-duals, the left-dual of $f$ is defined as

$f^{*}=\lambda_{X^{*}} \circ\left(\operatorname{ev}_{Y} \otimes \operatorname{id}_{X^{*}}\right) \circ \alpha_{Y^{*}, Y, X^{*}}^{-1} \circ\left(\operatorname{id}_{Y^{*}} \otimes\left(f \otimes \operatorname{id}_{X^{*}}\right)\right) \circ\left(\operatorname{id}_{Y^{*}} \otimes \operatorname{coev}_{X}\right) \circ \rho_{Y^{*}}^{-1}$.

A left-autonomous category is a monoidal category in which each object is equipped with a specified left-dual. 
Definition A.4. A braided monoidal category $(\mathcal{C}, \otimes, \mathbb{1}, \alpha, \lambda, \rho, \sigma)$ is a monoidal category $(\mathcal{C}, \otimes, \mathbb{1}, \alpha, \lambda, \rho)$ with natural isomorphisms $\sigma_{X, Y}: X \otimes Y \rightarrow Y \otimes X$ for all $X, Y \in|\mathcal{C}|$ that satisfy the two hexagon axioms

$$
\begin{aligned}
& \sigma_{X \otimes Y, Z}=\alpha_{Z, X, Y} \circ\left(\sigma_{X, Z} \otimes \operatorname{id}_{Y}\right) \circ \alpha_{X, Z, Y}^{-1} \circ\left(\operatorname{id}_{X} \otimes \sigma_{Y, Z}\right) \circ \alpha_{X, Y, Z}, \\
& \sigma_{X, Y \otimes Z}=\alpha_{Y, Z, X}^{-1} \circ\left(\operatorname{id}_{Y} \otimes \sigma_{X, Z}\right) \circ \alpha_{Y, X, Z} \circ\left(\sigma_{X, Y} \otimes \operatorname{id}_{Z}\right) \circ \alpha_{X, Y, Z}^{-1}
\end{aligned}
$$

for all $X, Y, Z \in|\mathcal{E}|$. The category is called symmetric monoidal if in addition

$$
\sigma_{Y, X} \circ \sigma_{X, Y}=\operatorname{id}_{X \otimes Y}
$$

for all $X, Y \in|\mathcal{E}|$.

Definition A.5. Let $(\mathcal{C}, \otimes, \mathbb{1}, \alpha, \lambda, \rho, \sigma)$ and $\left(\mathcal{C}^{\prime}, \otimes^{\prime}, \mathbb{1}^{\prime}, \alpha^{\prime}, \lambda^{\prime}, \rho^{\prime}, \sigma^{\prime}\right)$ be braided monoidal categories. A lax monoidal functor $\left(F, F_{X, Y}, F_{0}\right): \ell^{\prime} \ell^{\prime}$ is called braided if

$$
F \sigma_{X, Y} \circ F_{X, Y}=F_{Y, X} \circ \sigma_{F X, F Y}^{\prime}
$$

for all $X, Y \in|\mathcal{}|$.

\section{A.2. Abelian and semisimple categories}

Definition A.6. A category $\mathcal{C}$ is called Ab-enriched if it is enriched in the category $\mathbf{A b}$ of abelian groups, i.e. if $\operatorname{Hom}(X, Y)$ is an abelian group for all objects $X, Y \in|\mathcal{C}|$ and if the composition of morphisms is $\mathbb{Z}$-bilinear.

Let $k$ be a commutative ring. A category $C$ is called $k$-linear if it is enriched in ${ }_{k} \mathcal{M}$, the category of $k$-modules, i.e. if $\operatorname{Hom}(X, Y)$ is a $k$-module for all $X, Y \in|\mathcal{\ell}|$ and if the composition of morphisms is $k$-bilinear.

A functor $F: \mathcal{C} \rightarrow \ell^{\prime}$ between Ab-enriched (resp. $k$-linear) categories is called additive (resp. $k$-linear) if it induces homomorphisms of additive groups (resp. $k$-modules)

$$
\operatorname{Hom}(X, Y) \longrightarrow \operatorname{Hom}(F X, F Y)
$$

for all $X, Y \in|\mathcal{}|$.

Definition A.7. A monoidal category $(\mathcal{C}, \otimes, \mathbb{1}, \alpha, \lambda, \rho)$ is called Ab-enriched (resp. $k$-linear) if $\mathcal{C}$ is $\mathbf{A b}$-enriched (resp. $k$-linear) and if the tensor product of morphisms is $\mathbb{Z}$-bilinear (resp. $k$-bilinear).

Definition A.8. An additive category is an Ab-enriched category that has a terminal object and all binary products. A preabelian category is an Ab-enriched category that has all finite limits. An abelian category is a preabelian category in which every monomorphism is a kernel and in which every epimorphism is a cokernel.

Definition A.9. Let $\mathcal{C}$ be a $k$-linear category, $k$ a field. 
(1) An object $X \in|\mathcal{C}|$ is called simple if $\operatorname{End}(X) \cong k$ are isomorphic as $k$-modules.

(2) The category $\mathcal{C}$ is called split semisimple if there exists a family $\left\{V_{j}\right\}_{j \in I}$ of objects $V_{j} \in|\mathcal{E}|, I$ some index set, such that

(a) $V_{j}$ is simple for all $j \in I$.

(b) $\operatorname{Hom}\left(V_{j}, V_{\ell}\right)=\{0\}$ for all $j, \ell \in I$ for which $j \neq \ell$.

(c) For each object $X \in|\ell|$, there is a finite sequence $j_{1}^{(X)}, \ldots, j_{n^{X}}^{(X)} \in I$, $n^{X} \in \mathbb{N}_{0}$, and morphisms $\imath_{\ell}^{(X)}: V_{j_{\ell}} \rightarrow X$ and $\pi_{\ell}^{(X)}: X \rightarrow V_{j_{\ell}}$ such that

$$
\operatorname{id}_{X}=\sum_{\ell=1}^{n^{X}} t_{\ell}^{X} \circ \pi_{\ell}^{X} .
$$

and

$$
\pi_{\ell}^{X} \circ \imath_{m}^{X}= \begin{cases}\operatorname{id}_{V_{j_{\ell}}} & \text { if } \ell=m, \\ 0, & \text { else. }\end{cases}
$$

(3) The category is called finitely split semisimple if it is split semisimple with a finite index set $I$ in condition (3).

When we speak of split semisimple monoidal categories, we do not require the monoidal unit $\mathbb{1}$ to be simple. Note that in a split semisimple autonomous category, if an object $X$ is simple, then so is its dual.

\section{References}

[1] B. Bakalov and A. Kirillov, Lectures on tensor categories and modular functors. Amer. Math. Soc., Providence (RI), 2001. MR 1797619 Zbl 0965.18002

[2] G. Böhm, F. Nill, and K. Szlachányi, Weak Hopf algebras I. Integral theory and $C^{*}$-structure. J. Algebra 221 (1999), 385-438. MR 1726707 Zbl 0949.16037

[3] G. Böhm and K. Szlachányi, Weak Hopf algebras II. Representation theory, dimensions, and the Markov trace. J. Algebra 233 (2000), 156-212. MR 1793595 Zbl 0980.16028

[4] P. Etingof, D. Nikshych, and V. Ostrik, On fusion categories. Ann. of Math. (2) 162 (2005), 581-642. MR 2183279 Zbl 1125.16025

[5] T. Hayashi, Face algebras and unitarity of $S U(N)_{L}$-TQFT. Comm. Math. Phys. 203 (1999), 211-247. MR 1695168 Zbl 1055.81033

[6] T. Hayashi, A canonical Tannaka duality for finite semisimple tensor categories. Preprint 1999. arXiv:math/9904073

[7] M. Jimbo, T. Miwa, and M. Okado, Solvable lattice models related to the vector representation of classical simple Lie algebras. Comm. Math. Phys. 116 (1988), 507-525. MR 0937773 Zbl 0642.17016 
[8] L. H. Kauffman and S. L. Lins, Temperly-Lieb recoupling theory and invariants of 3-manifolds. Princeton University Press, Princeton (NJ), 1994. MR 1280463 Zbl 0821.57003

[9] L. Kadison and K. Szlachányi, Bialgebroid actions on depth two extensions and duality. Adv. Math. 179 (2003), 75-121. MR 2004729 Zbl 1049.16022

[10] S. Mac Lane, Categories for the working mathematician. Springer Verlag, New York etc., 1971. MR 0354798 Zbl 0232.18001

[11] M. B. McCurdy, Tannaka reconstruction of weak Hopf algebras in arbitrary monoidal categories. Preprint 2009. arXiv:0903.0208.

[12] E. Müller, Cosemisimple Hopf algebras and the FRT construction. Comm. Alg. 29 (2001), 4377-4394. MR 1854140 Zbl 0995.17005

[13] D. Nikshych, On the structure of weak Hopf algebras. Adv. Math. 170 (2002), 257-286. MR 1932332 Zbl 1010.16041

[14] A. Ocneanu, Quantized groups, string algebras and Galois theory for algebras. In D. E. Evans and M. Takesaki (eds), Operator algebras and applications. Volume II: Mathematical physics and subfactors. (Papers presented at a UK-US joint seminar on operator algebras held during 20-25 July 1987 at Warwick, UK). Cambridge University Press, Cambridge (U.K.), 1988, 119-172. MR 0996454 Zbl 0696.46048

[15] V. Ostrik, Module categories, weak Hopf algebras and modular invariants. Transform. Groups 8, No. 2 (2003), 177-206. MR 1976459 Zbl 1044.18004

[16] H. Pfeiffer, Tannaka-Kre ̌n reconstruction and a characterization of modular tensor categories. J. Algebra 321 (2009), 3714-3763. MR 2517811 Zbl 1208.18005

[17] H. Pfeiffer, Finitely semisimple spherical categories and modular categories are self-dual. Adv. Math. 221 (2009), 1608-1652. MR 2522429 Zbl 1173.16020

[18] N. Yu. Reshetikhin, L. A. Takhtajan, and L. D. Faddeev, Quantization of Lie groups and Lie algebras. Leningrad Math. J. 1 (1990), 193-225. MR 1015339 Zbl 0715.17015

[19] N. Yu. Reshetikhin and V. G. Turaev, Ribbon graphs and their invariants derived from quantum groups. Comm. Math. Phys. 127 (1990), 1-26. MR 1036112 Zbl 0768.57003

[20] P. Schauenburg, Weak Hopf algebras and quantum groupoids. In P. M. Hajac (ed.) et al., Noncommutative geometry and quantum groups. Proceedings of the Banach Center school/conference (Warsaw, 2001). Polish Academy of Sciences, Warsaw, 2003, 171-188. MR 2024429 Zbl 1064.16041

[21] P. Schauenburg, Tannaka duality for arbitrary Hopf algebras. Reinhard Fischer Verlag, München, 1992. MR 1623637 Zbl 0830.16029

[22] K. Szlachányi, Adjointable monoidal functors and quantum groupoids. In S. Caenepeel (ed.) et al., Hopf algebras in noncommutative geometry and physics. Proceedings of the conference on Hopf algebras and quantum groups, Brussels, Belgium, May 28-June 1, 2002. Marcel Dekker, New York, 2005, 291-307. MR 2106937 Zbl 1064.18008

[23] V. G. Turaev, Quantum invariants of knots and 3-manifolds. Walter de Gruyter, Berlin, 1994. MR 1292673 Zbl 0812.57003

[24] P. Vecsernyés, Larson-Sweedler theorem and the role of grouplike elements in weak Hopf algebras. J. Algebra 270 (2003), 471-520. MR 2019628 Zbl 1056.16034 
Received March 26, 2010

H. Pfeiffer, Department of Mathematics, The University of British Columbia, 1984 Mathematics Road, Vancouver, BC, V2T 1Z2, Canada

E-mail: pfeiffer@math.ubc.ca 